ESAIM: M2AN 47 (2013) 961-985

DOI: $10.1051 / \mathrm{m} 2 \mathrm{an} / 2012053$
ESAIM: Mathematical Modelling and Numerical Analysis

www.esaim-m2an.org

\title{
MATHEMATICAL MODEL OF BLOOD FLOW IN AN ANATOMICALLY DETAILED ARTERIAL NETWORK OF THE ARM
}

\author{
Sansuke M. Watanabe ${ }^{1,2,3}$, Pablo J. Blanco ${ }^{2,3}$ and Raúl A. Feijóo ${ }^{2,3}$
}

\begin{abstract}
A distributed-parameter (one-dimensional) anatomically detailed model for the arterial network of the arm is developed in order to carry out hemodynamics simulations. This work focuses on the specific aspects related to the model set-up. In this regard, stringent anatomical and physiological considerations have been pursued in order to construct the arterial topology and to provide a systematic estimation of the involved parameters. The model comprises 108 arterial segments, with 64 main arteries and 44 perforator arteries, with lumen radii ranging from $0.24 \mathrm{~cm}$ - axillary artery- to $0.018 \mathrm{~cm}-$ perforator arteries. The modeling of blood flow in deformable vessels is governed by a well-known set of hyperbolic partial differential equations that accounts for mass and momentum conservation and a constitutive equation for the arterial wall. The variational formulation used to solve the problem and the related numerical approach are described. The model rendered consistent pressure and flow rate outputs when compared with patient records already published in the literature. In addition, an application to dimensionally-heterogeneous modeling is presented in which the developed arterial network is employed as an underlying model for a three-dimensional geometry of a branching point to be embedded in order to perform local analyses.
\end{abstract}

Mathematics Subject Classification. 76Z05, 92C10, 92C30.

Received December 22, 2011.

Published online June 13, 2013.

\section{INTRODUCTION}

Since its introduction some decades ago, one-dimensional (1D) modeling of the blood flow in deformable vessels has proven to be a simple and effective approach to simulate the hemodynamics in the larger arteries of the body $[4,25]$. Several topological descriptions of the main arteries have been presented elsewhere, rendering useful information and consistent results with medical records and experimental data $[2,38,43,48]$.

In view of the large amount of data involved in the set-up of the model, previous works addressed the topology of the arterial network from a simplified point of view, that is by removing smaller arteries and simplifying connectivity among vessels as well as their relation with vascular territories. Nevertheless, there are

\footnotetext{
Keywords and phrases. Hemodynamics, anatomical model, vascular territories, numerical simulation.

1 Universidade Federal Rural de Pernambuco, Unidade Acadêmica de Garanhuns, Garanhuns, Brazil. sansuke@uag.ufrpe.br

2 Laboratório Nacional de Computação Científica, LNCC, Petrópolis, Brazil. sansuke@lncc.br;pjblanco@lncc.br;

3 Instituto Nacional de Ciência e Tecnologia em Medicina Assistida por Computação Científica, INCT-MACC, Petrópolis, Brazil.
} 

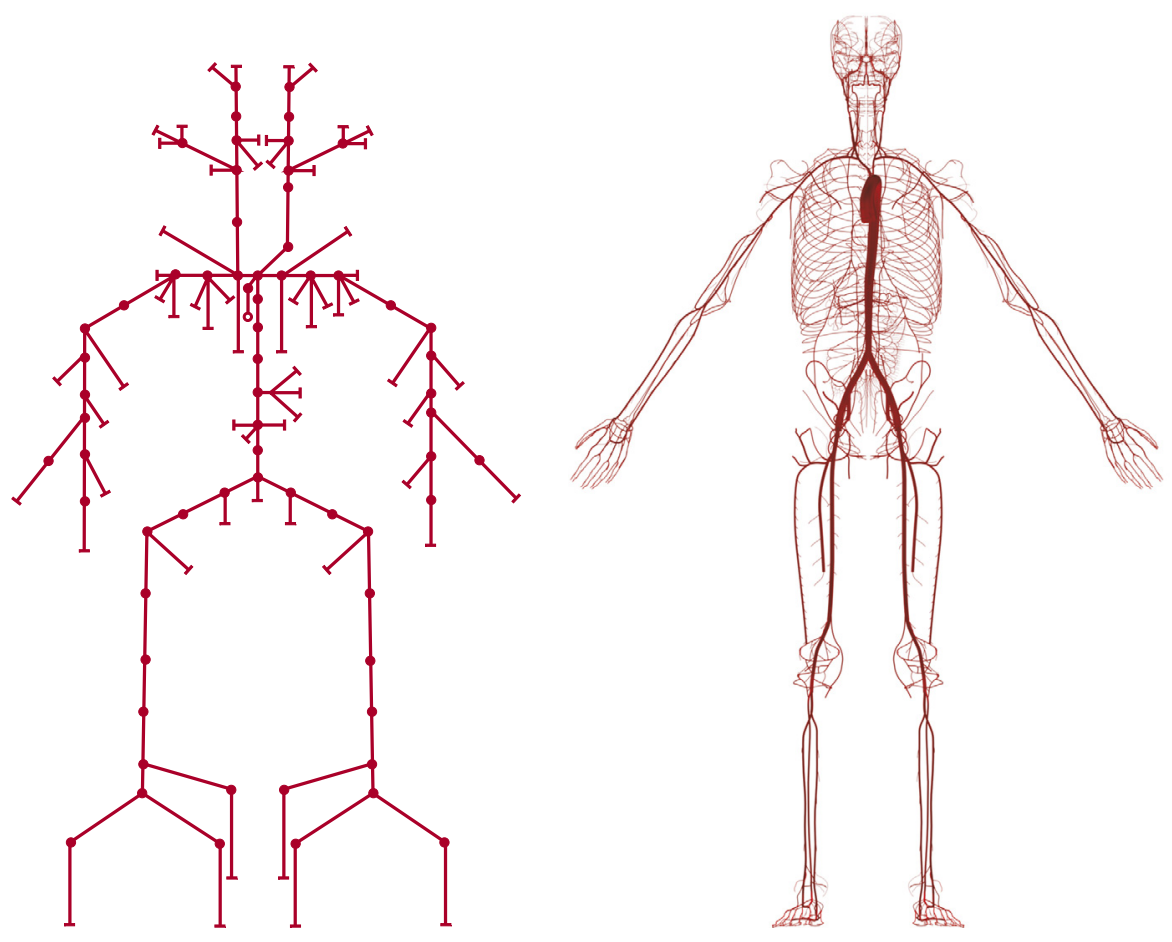

FiguRE 1. Simplified and anatomically detailed model for the arterial network. The former is taken from [4], and the later is the basis for the model presented in this work.

specific applications which require to take into consideration the true topology of the cardiovascular system, redundancies in the circulation, anastomoses, etc. in order to account for a proper blood flow distribution as well as to be able to model certain physiological and/or pathophysiological scenarios. Surgical procedures like radial harvesting for coronary revascularization and its impact on the blood flow supply to the arteries in the hand is a clear example of medical interest [27]. Other instances are procedures related to reconstructive surgery, in which knowing the blood supply to the different vascular territories may be of potential clinical relevance in order to perform tissue transplantation sucessfully [40,49].

The previous paragraph sets clear examples to motivate the development of detailed anatomical models of the arterial system to perform blood flow simulations. Available models of the arterial network topology look like the one shown in Figure 1-left (incorporating the main 128 arterial vessels), while when looking into catalogued anatomical data it is possible to identify an arterial network topology as the one shown in Figure 1-right. The former is referred to as simplified model, and in this case it corresponds to the model presented in [4] and used extensively in previous works [6], and the later is referred to as anatomically detailed model, and comprises the foundational topology for the model developed in the present work.

In the present work we focus on the arterial functioning and blood supply to the vascular territories of the arm. The novelty of the present work is the set-up of an anatomically detailed model for the arterial network of the arm following stringent anatomical and physiological considerations. The final goal is to establish the data infrastructure (geometrical and mechanical parameters) to be able to perform 1D blood flow simulations. Existing models of the cardiovascular system account for the main arteries of the system. For example, concerning the arteries of the arm, in $[4,28,43]$ the axillary, brachial, radial and ulnar arteries are incorporated. At most, the interosseous artery is also included, an important branch of the ulnar artery. In turn, the model developed in the present contribution comprises 108 arteries whose lumen radii range from $0.24 \mathrm{~cm}$ - axillary artery - to $0.018 \mathrm{~cm}$ 
- perforator arteries (see Figs. 1 and 4). This includes the main 40 arterial vessels of the upper arm, the forearm and the hand reported in the anatomical literature [16]. Taking into account repeated occurrences (common palmar digital arteries, for instance) the model grows up to 64 arterial segments altogether. In addition, arteries that perform tissue blood supply, called perforator arteries, are located along the network in order to derive the blood flow to the different vascular territories through the peripheral beds. In total, 44 perforator arteries were included, totalizing 108 arterial segments. The terminal vessels are coupled to lumped models that take into account the effect of the peripheral vasculature not considered in the model. A total of 67 terminals is placed at the most distal sites of the network. In view of the small size of the terminal arteries, of the order of $0.018 \mathrm{~cm}$ of lumen radii, purely resistive lumped models have been considered. Resistive parameters of terminal models were estimated by taking into account the muscular resistance of the associated vascular territory fed by the corresponding perforator artery. This model of the vasculature of the arm has been used in [12] to develop an algorithm for the fine calibration of outflow conditions.

The organization of the work is as follows: Section 1 presents the governing equations for the arterial blood flow as well as for the terminal sites. In Section 2 the variational formulation and the numerical approach used to obtain approximate solutions to the problem are presented. In Section 3 the criteria used to perform the parameter set-up is discussed. The anatomical and physiological considerations are outlined in Section 4, while in Section 5 some results obtained with this model are shown and compared with available patient records. In Section 6 the application of the arterial network to dimensionally-heterogeneous modeling is presented. Final remarks are drawn in Section 7.

\section{Mathematical MODEL}

\subsection{Vessel model}

A 1D representation of the blood flow in a compliant vessel is governed by a set of equations which can be derived from the full Navier-Stokes equations [25]. Under certain geometrical and kinematical hypotheses, such a set of non-linear hyperbolic partial differential equations can be written as

$$
\frac{\partial A}{\partial t}+\frac{\partial Q}{\partial x}=0
$$

and

$$
\frac{\partial Q}{\partial t}+\frac{\partial}{\partial x}\left(\alpha \frac{Q^{2}}{A}\right)=-\frac{A}{\rho} \frac{\partial P}{\partial x}-2 \pi R \tau
$$

where $A=A(x, t)$ is the arterial lumen cross sectional area and $R=R(x, t)$ is the corresponding radius, $Q=Q(x, t)$ is the flow rate, $P=P(x, t)$ is the mean pressure, $\rho$ is the blood density, $\tau=\tau(x, t)$ is the wall shear stress and $\alpha=\alpha(x, t)$ is the convective aceleration parameter defined by

$$
\alpha=\frac{A}{Q^{2}} \int_{A} u^{2} \mathrm{~d} A
$$

where $u=u(r, x, t)$ is the axial velocity written in terms of the axial and radial coordinates, $x$ and $r$ respectively. This parameter varies in the range $[1,4 / 3]$, where $\alpha=1$ leads to a plane velocity profile and $\alpha=4 / 3$ to a parabolic one. In this work, we have considered $\alpha=1$. In addition, $\tau=\mu d u /\left.d r\right|_{r=R}=4 \mu Q /\left(\pi R^{3}\right)$, where $\mu$ is the blood viscosity. Equations (1.1) and (1.2) stand for mass and momentum conservation, respectively. In order to close the system of equations a constitutive equation relating the mean pressure $P$ to the cross sectional area $A$ is required. In the present contribution a simple pure elastic wall behavior is assumed, which reads

$$
P=P_{o}+\frac{E h}{R_{o}}\left(\sqrt{\frac{A}{A_{o}}}-1\right)
$$


where $E$ is the effective Young's modulus, $h$ is the arterial wall thickness and the subscript " $o$ " means that such quantities are evaluated at a reference pressure $P_{o}$. Summarizing, the parameters required by each arterial vessel are $\left\{E, h, R_{o}\right\}$, while other general parameters are $\left\{\rho, \mu, P_{o}\right\}$.

\subsection{Bifurcation model}

At bifurcation sites continuity of mass is required, while continuity of pressure is considered. Then, for $V$ vessels arriving at a bifurcation we have the following equations

$$
\sum_{i=1}^{V} Q_{i}=0,
$$

for mass conservation and

$$
P_{1}=P_{i} \quad \forall i=2, \ldots, V,
$$

for continuity of pressure. Continuity of pressure has been tested and provided accurate results in the physiological regime against experimental data [1].

\subsection{Terminal model}

Lumped parameter terminal models are incorporated in the mathematical description to account for the remaining part of the vasculature, that is smaller arteries, arterioles and capillaries. From a physiological point of view, terminal models govern the blood flow distribution in the arterial network, while from a mathematical perspective they act as boundary conditions relating the pressure to the flow rate at a certain terminal site. Usually, the well-known three-element windkessel model is employed in 1D simulations [43], which establishes a relation between pressure and flow rate through an ordinary differential equation. In the present, in view of the small size of distal-most arteries, we have adopted a purely resistive model. Therefore, we have

$$
Q=\frac{\Delta P}{\mathcal{R}}
$$

where $Q$ is the flow rate through the terminal, $\Delta P$ is the pressure drop in the peripheral bed and $\mathcal{R}$ is the terminal resistance, which is the only model parameter needed at the peripheral sites.

\section{NumericAl METHOD}

Let us begin reducing equations (1.2) and (1.4) to a single expression. So, by the chain rule, we can wite

$$
\frac{\partial P}{\partial x}=\frac{\partial P}{\partial A} \frac{\partial A}{\partial x}+\sum_{i} \frac{\partial P}{\partial \lambda_{i}} \frac{\partial \lambda_{i}}{\partial x}
$$

where $\lambda_{i} \in\left\{E, h, R_{o}, P_{o}\right\}$. The speed of sound $c$ in the vessel is defined as

$$
c=\sqrt{\frac{A}{\rho} \frac{\partial P}{\partial A}} .
$$

In this way, equations (1.2) and (1.4) can be reduced to a single equation in terms of $A$ and $Q$ and rewritten as

$$
\frac{\partial Q}{\partial t}+\frac{\partial}{\partial x}\left(\alpha \frac{Q^{2}}{A}\right)+c^{2} \frac{\partial A}{\partial x}=g
$$

where

$$
g=-\frac{A}{\rho} \sum_{i} \frac{\partial P}{\partial \lambda_{i}} \frac{\partial \lambda_{i}}{\partial x}-2 \pi R \tau .
$$


Now, we can write equations (1.1) and (2.3) as follows [20,22]

$$
\frac{\partial \mathbf{U}}{\partial t}+\mathbf{H}(\mathbf{U}) \frac{\partial \mathbf{U}}{\partial x}=\mathbf{B}
$$

where $\mathbf{U}=[A Q]^{T}, \mathbf{B}=\left[\begin{array}{ll}0 & g\end{array}\right]^{T}$ and

$$
\mathbf{H}(\mathbf{U})=\left[\begin{array}{cc}
0 & 1 \\
-\alpha \frac{Q^{2}}{A^{2}}+c^{2} & 2 \alpha \frac{Q}{A}
\end{array}\right] .
$$

The eigenvalues of $\mathbf{H}$ are

$$
\begin{aligned}
& f^{+}=\alpha \frac{Q}{A}+\sqrt{\alpha(\alpha-1) \frac{Q^{2}}{A^{2}}+c^{2}}, \\
& f^{-}=\alpha \frac{Q}{A}-\sqrt{\alpha(\alpha-1) \frac{Q^{2}}{A^{2}}+c^{2}} .
\end{aligned}
$$

Clearly, $f^{ \pm} \in \mathbb{R}$ for all values of $A$ and $Q$. Thus, it means that the system (2.5) is hyperbolic and $f^{+}=$ $f^{+}(t, x, A, Q), f^{-}=f^{-}(t, x, A, Q)$ are the characteristic speeds of the system. Moreover, this system of equations can be recasted along its characteristic lines and written in an alternative form

$$
\begin{aligned}
& \frac{D Q}{D t}-f^{-} \frac{D A}{D t}=g \text { along } \dot{x}=f^{+}[t, x(t), A(t, x(t)), Q(t, x(t))], \\
& \frac{D Q}{D t}-f^{+} \frac{D A}{D t}=g \text { along } \dot{x}=f^{-}[t, x(t), A(t, x(t)), Q(t, x(t))],
\end{aligned}
$$

where notation $D(\cdot) / D t$ stands for the total derivative along the corresponding characteristic line. Now, expanding the total derivatives in (2.9) and (2.10) we obtain

$$
\begin{aligned}
& \frac{\partial Q}{\partial t}+f^{+} \frac{\partial Q}{\partial x}-f^{-}\left(\frac{\partial A}{\partial t}+f^{+} \frac{\partial A}{\partial x}\right)=g \\
& \frac{\partial Q}{\partial t}+f^{-} \frac{\partial Q}{\partial x}-f^{+}\left(\frac{\partial A}{\partial t}+f^{-} \frac{\partial A}{\partial x}\right)=g .
\end{aligned}
$$

In order to attempt a numerical solution, we firstly perform an Euler's semi-implicit time discretization for equations (2.11) and (2.12), and then we define two residuals for each time step, which are given by

$$
\begin{aligned}
& R_{+}\left(A^{n+1}, Q^{n+1}\right)=\frac{Q^{n+1}-Q^{n}}{\Delta t}+\left.f^{+} \frac{\partial Q}{\partial x}\right|_{n+\theta}-f^{-}\left(\frac{A^{n+1}-A^{n}}{\Delta t}+\left.f^{+} \frac{\partial A}{\partial x}\right|_{n+\theta}\right)-g^{n+\theta}, \\
& R_{-}\left(A^{n+1}, Q^{n+1}\right)=\frac{Q^{n+1}-Q^{n}}{\Delta t}+\left.f^{-} \frac{\partial Q}{\partial x}\right|_{n+\theta}-f^{+}\left(\frac{A^{n+1}-A^{n}}{\Delta t}+\left.f^{-} \frac{\partial A}{\partial x}\right|_{n+\theta}\right)-g^{n+\theta},
\end{aligned}
$$

with $\frac{1}{2} \leq \theta \leq 1$ and $\left.(\cdot)\right|_{n+\theta}=\left.\theta(\cdot)\right|_{n+1}+\left.(1-\theta)(\cdot)\right|_{n}$. To reach the variational formulation that will be used as starting point for the numerical approximation, we make use of a least square approach. Consider a single arterial segment, then we define a cost functional given by

$$
\mathcal{J}\left(A^{n+1}, Q^{n+1}\right)=\frac{1}{2} \int_{0}^{L}\left(R_{+}^{2}+R_{-}^{2}\right) \mathrm{d} x,
$$


where $L$ is the lenght of the vessel. The associated set of variational equations is the following

$$
\begin{gathered}
\frac{\partial \mathcal{J}}{\partial A^{n+1}}=\left.\frac{\mathrm{d}}{\mathrm{d} \xi} \mathcal{J}\left(A^{n+1}+\xi \tilde{A}, Q^{n+1}\right)\right|_{\xi=0}=0, \\
\frac{\partial \mathcal{J}}{\partial Q^{n+1}}=\left.\frac{\mathrm{d}}{\mathrm{d} \xi} \mathcal{J}\left(A^{n+1}, Q^{n+1}+\xi \tilde{Q}\right)\right|_{\xi=0}=0 .
\end{gathered}
$$

So (2.16) and (2.17) yield the following problem: find $\left(A^{n+1}, Q^{n+1}\right) \in \mathcal{U}$ such that

$$
\begin{array}{r}
\int_{0}^{L}\left[R_{+} f^{-}\left(\tilde{A}+h^{+} \theta \frac{\partial \tilde{A}}{\partial x}\right)+R_{-} f^{+}\left(\tilde{A}+h^{-} \theta \frac{\partial \tilde{A}}{\partial x}\right)\right] \mathrm{d} x=0, \forall(\tilde{A}, 0) \in \mathcal{V} \\
\int_{0}^{L}\left[R_{+}\left(\tilde{Q}+h^{+} \theta \frac{\partial \tilde{Q}}{\partial x}\right)+R_{-}\left(\tilde{Q}+h^{-} \theta \frac{\partial \tilde{Q}}{\partial x}\right)\right] \mathrm{d} x=0, \forall(0, \tilde{Q}) \in \mathcal{V},
\end{array}
$$

where $\mathcal{U}$ is the set of admissible functions and $\mathcal{V}$ is its associated linear space of admissible variations, $h^{ \pm}=\Delta t f^{ \pm}$ is a stabilization parameter. Notice that the well-known numerical stabilization required by the problem is derived naturally from the semi-discrete (discrete in time) formulation. After inserting residuals $R_{ \pm}$given by (2.13) and (2.14) into variational equations (2.18) and (2.19) and expanding the (.) $\left.\right|_{n+\theta}$ notation, we rewrite the above system of nonlinear equations (whose unknowns are $A^{n+1}$ and $Q^{n+1}$ ) as follows

$$
\begin{aligned}
& C_{A A}\left(A^{n+1}, \tilde{A}\right)+C_{Q A}\left(Q^{n+1}, \tilde{A}\right)=b_{A}(\tilde{A}), \quad \forall(\tilde{A}, 0) \in \mathcal{V}, \\
& C_{A Q}\left(A^{n+1}, \tilde{Q}\right)+C_{Q Q}\left(Q^{n+1}, \tilde{Q}\right)=b_{Q}(\tilde{Q}), \quad \forall(0, \tilde{Q}) \in \mathcal{V},
\end{aligned}
$$

where

$$
\begin{aligned}
C_{A A}\left(A^{n+1}, \tilde{A}\right)= & \int_{0}^{L}-\left[\left(\frac{\left(f^{-}\right)^{2}}{\Delta t} A^{n+1}+\theta\left(f^{-}\right)^{2} f^{+} \frac{\partial}{\partial x} A^{n+1}\right)\left(\tilde{A}+\theta h^{+} \frac{\partial \tilde{A}}{\partial x}\right)\right. \\
+ & \left.\left(\frac{\left(f^{+}\right)^{2}}{\Delta t} A^{n+1}+\theta\left(f^{+}\right)^{2} f^{-} \frac{\partial}{\partial x} A^{n+1}\right)\left(\tilde{A}+\theta h^{-} \frac{\partial \tilde{A}}{\partial x}\right)\right] \mathrm{d} x \\
C_{Q A}\left(Q^{n+1}, \tilde{A}\right)= & \int_{0}^{L}\left[\left(\frac{f^{-}}{\Delta t} Q^{n+1}+\theta f^{+} f^{-} \frac{\partial}{\partial x} Q^{n+1}\right)\left(\tilde{A}+\theta h^{+} \frac{\partial \tilde{A}}{\partial x}\right)\right. \\
& \left.+\left(\frac{f^{+}}{\Delta t} Q^{n+1}+\theta f^{-} f^{+} \frac{\partial}{\partial x} Q^{n+1}\right)\left(\tilde{A}+\theta h^{-} \frac{\partial \tilde{A}}{\partial x}\right)\right] \mathrm{d} x \\
C_{A Q}\left(A^{n+1}, \tilde{Q}\right)= & \int_{0}^{L}-\left[\left(\frac{f^{-}}{\Delta t} A^{n+1}+\theta f^{-} f^{+} \frac{\partial}{\partial x} A^{n+1}\right)\left(\tilde{Q}+\theta h^{+} \frac{\partial \tilde{Q}}{\partial x}\right)\right. \\
+ & \left.\left(\frac{f^{+}}{\Delta t} A^{n+1}+\theta f^{+} f^{-} \frac{\partial}{\partial x} A^{n+1}\right)\left(\tilde{Q}+\theta h^{-} \frac{\partial \tilde{Q}}{\partial x}\right)\right] \mathrm{d} x \\
C_{Q Q}\left(Q^{n+1}, \tilde{Q}\right)= & \int_{0}^{L}\left[\left(\frac{Q^{n+1}}{\Delta t}+\theta f^{+} \frac{\partial}{\partial x} Q^{n+1}\right)\left(\tilde{Q}+\theta h^{+} \frac{\partial \tilde{Q}}{\partial x}\right)\right. \\
& \left.+\left(\frac{Q^{n+1}}{\Delta t}+\theta f^{-} \frac{\partial}{\partial x} Q^{n+1}\right)\left(\tilde{Q}+\theta h^{-} \frac{\partial \tilde{Q}}{\partial x}\right)\right] \mathrm{d} x
\end{aligned}
$$




$$
\begin{aligned}
b_{A}(\tilde{A})= & \int_{0}^{L}\left[\left(-\frac{\left(f^{-}\right)^{2}}{\Delta t} A^{n}+(1-\theta)\left(f^{-}\right)^{2} f^{+} \frac{\partial}{\partial x} A^{n}+\frac{f^{-}}{\Delta t} Q^{n}-(1-\theta) f^{+} f^{-} \frac{\partial}{\partial x} Q^{n}\right.\right. \\
& \left.+f^{-}\left(\theta g^{n+1}+(1-\theta) g^{n}\right)\right)\left(\tilde{A}+\theta h^{+} \frac{\partial \tilde{A}}{\partial x}\right) \\
& +\left(-\frac{\left(f^{+}\right)^{2}}{\Delta t} A^{n}+(1-\theta)\left(f^{+}\right)^{2} f^{-} \frac{\partial}{\partial x} A^{n}+\frac{f^{+}}{\Delta t} Q^{n}-(1-\theta) f^{-} f^{+} \frac{\partial}{\partial x} Q^{n}\right. \\
& \left.\left.+f^{+}\left(\theta g^{n+1}+(1-\theta) g^{n}\right)\right)\left(\tilde{A}+\theta h^{-} \frac{\partial \tilde{A}}{\partial x}\right)\right] \mathrm{d} x,
\end{aligned}
$$

and

$$
\begin{aligned}
b_{Q}(\tilde{Q})= & \int_{0}^{L}\left[\left(-\frac{f^{-}}{\Delta t} A^{n}+(1-\theta) f^{-} f^{+} \frac{\partial}{\partial x} A^{n}+\frac{Q^{n}}{\Delta t}-(1-\theta) f^{+} \frac{\partial}{\partial x} Q^{n}\right.\right. \\
& \left.\left.+\theta g^{n+1}+(1-\theta) g^{n}\right)\right)\left(\tilde{Q}+\theta h^{+} \frac{\partial \tilde{Q}}{\partial x}\right) \\
& +\left(-\frac{f^{+}}{\Delta t} A^{n}+(1-\theta) f^{+} f^{-} \frac{\partial}{\partial x} A^{n}+\frac{Q^{n}}{\Delta t}-(1-\theta) f^{-} \frac{\partial}{\partial x} Q^{n}\right. \\
& \left.\left.\left.+\theta g^{n+1}+(1-\theta) g^{n}\right)\right)\left(\tilde{Q}+\theta h^{-} \frac{\partial \tilde{Q}}{\partial x}\right)\right] \mathrm{d} x .
\end{aligned}
$$

In order to linearize the system given by $(2.20)$ and (2.21) we introduce a fixed-point method so that indexes $k+1$ and $k$ stand for current and previous iterations, respectively. In this way, we obtain the following system of linear variational equations in the unknowns $A^{n+1, k+1}$ and $Q^{n+1, k+1}$

$$
\begin{aligned}
& C_{A A}^{k}\left(A^{n+1, k+1}, \tilde{A}\right)+C_{Q A}^{k}\left(Q^{n+1, k+1}, \tilde{A}\right)=b_{A}^{k}(\tilde{A}), \quad \forall(\tilde{A}, 0) \in \mathcal{V}, \\
& C_{Q A}^{k}\left(A^{n+1, k+1}, \tilde{Q}\right)+C_{Q Q}^{k}\left(Q^{n+1, k+1}, \tilde{Q}\right)=b_{Q}^{k}(\tilde{Q}), \quad \forall(0, \tilde{Q}) \in \mathcal{V},
\end{aligned}
$$

with

$$
\begin{aligned}
C_{A A}^{k}\left(A^{n+1, k+1}, \tilde{A}\right)= & \int_{0}^{L}-\left[\left(\frac{\left[\left(f^{-}\right)^{2}\right]^{n+1, k}}{\Delta t} A^{n+1, k+1}+\theta\left[\left(f^{-}\right)^{2} f^{+}\right]^{n+1, k} \frac{\partial}{\partial x} A^{n+1, k+1}\right)\left(\tilde{A}+\theta\left[h^{+}\right]^{n+1, k} \frac{\partial \tilde{A}}{\partial x}\right)\right. \\
& \left.+\left(\frac{\left[\left(f^{+}\right)^{2}\right]^{n+1, k}}{\Delta t} A^{n+1, k+1}+\theta\left[\left(f^{+}\right)^{2} f^{-}\right]^{n+1, k} \frac{\partial}{\partial x} A^{n+1, k+1}\right)\left(\tilde{A}+\theta\left[h^{-}\right]^{n+1, k} \frac{\partial \tilde{A}}{\partial x}\right)\right] \mathrm{d} x \\
C_{Q A}^{k}\left(Q^{n+1, k+1}, \tilde{A}\right)= & \int_{0}^{L}\left[\left(\frac{\left[f^{-}\right]^{n+1, k}}{\Delta t} Q^{n+1, k+1}+\theta\left[f^{+} f^{-}\right]^{n+1, k} \frac{\partial}{\partial x} Q^{n+1, k+1}\right)\left(\tilde{A}+\theta\left[h^{+}\right]^{n+1, k} \frac{\partial \tilde{A}}{\partial x}\right)\right. \\
& \left.+\left(\frac{\left[f^{+}\right]^{n+1, k}}{\Delta t} Q^{n+1, k+1}+\theta\left[f^{-} f^{+}\right]^{n+1, k} \frac{\partial}{\partial x} Q^{n+1, k+1}\right)\left(\tilde{A}+\theta\left[h^{-}\right]^{n+1, k} \frac{\partial \tilde{A}}{\partial x}\right)\right] \mathrm{d} x, \quad(2.31)
\end{aligned}
$$




$$
\begin{aligned}
& C_{A Q}^{k}\left(A^{n+1, k+1}, \tilde{Q}\right)=\int_{0}^{L}-\left[\left(\frac{\left[f^{-}\right]^{n+1, k}}{\Delta t} A^{n+1, k+1}+\theta\left[f^{-} f^{+}\right]^{n+1, k} \frac{\partial}{\partial x} A^{n+1, k+1}\right)\left(\tilde{Q}+\theta\left[h^{+}\right]^{n+1, k} \frac{\partial \tilde{Q}}{\partial x}\right)\right. \\
& \left.+\left(\frac{\left[f^{+}\right]^{n+1, k}}{\Delta t} A^{n+1, k+1}+\theta\left[f^{+} f^{-}\right]^{n+1, k} \frac{\partial}{\partial x} A^{n+1, k+1}\right)\left(\tilde{Q}+\theta\left[h^{-}\right]^{n+1, k} \frac{\partial \tilde{Q}}{\partial x}\right)\right] \mathrm{d} x, \\
& C_{Q Q}^{k}\left(Q^{n+1, k+1}, \tilde{Q}\right)=\int_{0}^{L}\left[\left(\frac{Q^{n+1, k+1}}{\Delta t}+\theta\left[f^{+}\right]^{n+1, k} \frac{\partial}{\partial x} Q^{n+1, k+1}\right)\left(\tilde{Q}+\theta\left[h^{+}\right]^{n+1, k} \frac{\partial \tilde{Q}}{\partial x}\right)\right. \\
& \left.+\left(\frac{Q^{n+1, k+1}}{\Delta t}+\theta\left[f^{-}\right]^{n+1, k} \frac{\partial}{\partial x} Q^{n+1, k+1}\right)\left(\tilde{Q}+\theta\left[h^{-}\right]^{n+1, k} \frac{\partial \tilde{Q}}{\partial x}\right)\right] \mathrm{d} x, \\
& b_{A}^{k}(\tilde{A})=\int_{0}^{L}\left[\left(-\frac{\left[\left(f^{-}\right)^{2}\right]^{n+1, k}}{\Delta t} A^{n}+(1-\theta)\left[\left(f^{-}\right)^{2} f^{+}\right]^{n+1, k} \frac{\partial}{\partial x} A^{n}\right.\right. \\
& +\frac{\left[f^{-}\right]^{n+1, k}}{\Delta t} Q^{n}-(1-\theta)\left[f^{+} f^{-}\right]^{n+1, k} \frac{\partial}{\partial x} Q^{n} \\
& \left.+\left[f^{-}\right]^{n+1, k}\left(\theta g^{n+1, k}+(1-\theta) g^{n}\right)\right)\left(\tilde{A}+\theta\left[h^{+}\right]^{n+1, k} \frac{\partial \tilde{A}}{\partial x}\right) \\
& +\left(-\frac{\left[\left(f^{+}\right)^{2}\right]^{n+1, k}}{\Delta t} A^{n}+(1-\theta)\left[\left(f^{+}\right)^{2} f^{-}\right]^{n+1, k} \frac{\partial}{\partial x} A^{n}\right. \\
& +\frac{\left[f^{+}\right]^{n+1, k}}{\Delta t} Q^{n}-(1-\theta)\left[f^{-} f^{+}\right]^{n+1, k} \frac{\partial}{\partial x} Q^{n} \\
& \left.\left.+\left[f^{+}\right]^{n+1, k}\left(\theta g^{n+1, k}+(1-\theta) g^{n}\right)\right)\left(\tilde{A}+\theta\left[h^{-}\right]^{n+1, k} \frac{\partial \tilde{A}}{\partial x}\right)\right] \mathrm{d} x
\end{aligned}
$$

and

$$
\begin{aligned}
b_{Q}^{k}(\tilde{Q})= & \int_{0}^{L}\left[\left(-\frac{\left[f^{-}\right]^{n+1, k}}{\Delta t} A^{n}+(1-\theta)\left[f^{-} f^{+}\right]^{n+1, k} \frac{\partial}{\partial x} A^{n}+\frac{Q^{n}}{\Delta t}-(1-\theta)\left[f^{+}\right]^{n+1, k} \frac{\partial}{\partial x} Q^{n}\right.\right. \\
& \left.\left.+\theta g^{n+1, k}+(1-\theta) g^{n}\right)\right)\left(\tilde{Q}+\theta\left[h^{+}\right]^{n+1, k} \frac{\partial \tilde{Q}}{\partial x}\right) \\
& +\left(-\frac{\left[f^{+}\right]^{n+1, k}}{\Delta t} A^{n}+(1-\theta)\left[f^{+} f^{-}\right]^{n+1, k} \frac{\partial}{\partial x} A^{n}+\frac{Q^{n}}{\Delta t}-(1-\theta)\left[f^{-}\right]^{n+1, k} \frac{\partial}{\partial x} Q^{n}\right. \\
& \left.\left.\left.+\theta g^{n+1, k}+(1-\theta) g^{n}\right)\right)\left(\tilde{Q}+\theta\left[h^{-}\right]^{n+1, k} \frac{\partial \tilde{Q}}{\partial x}\right)\right] \mathrm{d} x .
\end{aligned}
$$

The finite element method is employed to create a finite-dimensional basis $\left\{\phi_{1}, \ldots, \phi_{N}\right\}$ to approximate equations (2.28) and (2.29) by using first order Lagrange polynomial interpolation. In addition, by applying Newton method in order to approximate the constitutive equation (1.4), we obtain the following linearized area-pressure relation

$$
P^{n+1, k+1}-\frac{E h}{2 R_{o} \sqrt{A_{o} A^{n+1, k}}} A^{n+1, k+1}=P^{n+1, k}-\frac{E h}{2 R_{o} \sqrt{A_{o} A^{n+1, k}}} A^{n+1, k} .
$$


This equation is introduced in the discrete formulation in a node-wise sense. Thus, the discrete problem yields a $3 N \times 3 N$ system of linear equations whose structure in blocks is given by

$$
\left(\begin{array}{ccc}
\mathrm{C}_{Q Q} & \mathrm{C}_{A Q} & 0 \\
\mathrm{C}_{Q A} & \mathrm{C}_{A A} & 0 \\
0 & \mathrm{~B}_{A P} & \mathrm{I}
\end{array}\right)\left(\begin{array}{l}
\mathrm{Q} \\
\mathrm{A} \\
\mathrm{P}
\end{array}\right)=\left(\begin{array}{c}
\mathrm{b}_{Q} \\
\mathrm{~b}_{A} \\
\mathrm{~b}_{P}
\end{array}\right)
$$

where $\mathbf{I}$ is the identity matrix and 0 is the matrix with null entries. In addition, for $i, j=1, \ldots, N$ it is $[\mathrm{Q}]_{j}=Q_{j}^{n+1, k+1},[\mathrm{~A}]_{j}=A_{j}^{n+1, k+1},[\mathrm{P}]_{j}=P_{j}^{n+1, k+1},\left[\mathrm{C}_{X Y}\right]_{i j}=C_{X Y}^{k}\left(\phi_{j}, \phi_{i}\right)$ and $\left[\mathrm{b}_{Y}\right]_{i}=b_{N}^{k}\left(\phi_{i}\right), X, Y \in\{Q, A\}$. Finally, it is

$$
\begin{array}{rr}
{\left[\mathrm{B}_{A P}\right]_{i j}=-\frac{E h}{2 R_{o} \sqrt{A_{o} A_{i}^{n+1, k}}} \delta_{i j}} & i, j=1, \ldots, N \\
{\left[\mathrm{~b}_{P}\right]_{i}=P_{i}^{n+1, k}-\frac{E h}{2 R_{o} \sqrt{A_{o} A_{i}^{n+1, k}}} A_{i}^{n+1, k}} & i=1, \ldots, N .
\end{array}
$$

\section{Parameter estimation}

In order to feed the governing equations, we need a set of geometrical and mechanical parameters for arterial vessels and terminals. Nevertheless, in most cases, the external diameters of arteries are the only information reported in the medical literature. Moreover, gathering the data related to the external diameters of all the main arteries turns to be an arduous task. We have carried out such task, therefore, from this point onwards we consider that the external arterial diameters are known, and then we discuss the approaches used to assess the internal radius, the wall thickness and the effective Young's modulus. Furthermore, there were few cases in which the external arterial diameters were not found in the literature. There, we used either the Murray's law or the magnitude order of surrounding vessels to estimate them.

\subsection{Wall thickness}

A table with 76 values of internal radii and their respective wall thickness for 128 arterial segments is reported in [4]. Hence, for each internal radius $r$, we evaluated the ratio $h / r$, where $h$ is the correspondent wall thickness. The 76 points were fitted by the curve given by

$$
\frac{h}{r}=a \mathrm{e}^{b r}+c \mathrm{e}^{d r}
$$

with $a=0.2802, b=-5.053, c=0.1324$ and $d=-0.1114$. The points and the fitted curve are shown in Figure 2.

Therefore, given the lumen radius $r$ for each artery, we use equation (3.1) to assess its wall thickness $h$.

\subsection{Internal radius}

As said, most of arterial calibers were found in morphometrical assessments whose purpose was to measure external diameters. However, we are concerned in the arterial lumen radius. Thus, denoting by $D$ the external diameter of an artery, we obtain the reference value for its internal radius $r$ by solving the following nonlinear equation

$$
2 r\left(1+\frac{h}{r}\right)=D
$$

where the ratio $h / r$ is given by expression (3.1). 


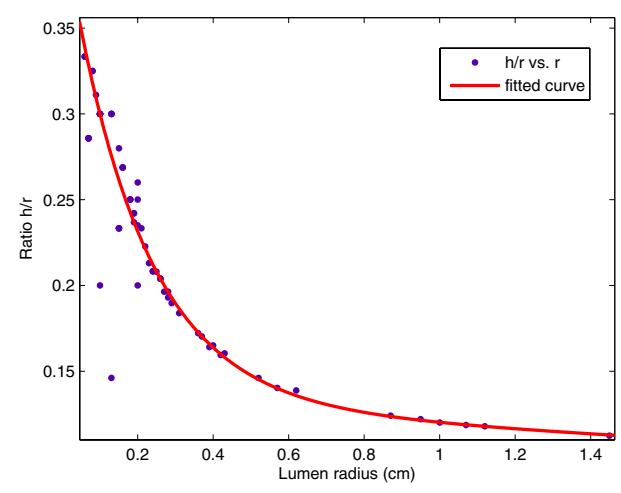

FiguRE 2. The ratio $h / r$ as a function of the lumen radius $r$.

\subsection{Elastic properties}

Based on empirical data, an exponential expression for the ratio $E h / r$ as a function of the lumen radius was provided in [34], where $E$ is the effective Young's modulus. So, this parameter $E$ can be written as a function of $(r, h)$, yielding

$$
E(r, h)=\frac{r}{h}\left(k_{1} \mathrm{e}^{k_{2} r}+k_{3}\right),
$$

where $k_{1}=1.4 \times 10^{7}$ dyn $\mathrm{cm}^{-2}, k_{2}=-22.53 \mathrm{~cm}^{-1}$ and $k_{3}=6.055 \times 10^{5} \mathrm{dyn} \mathrm{cm}^{-2}$ are constants. However, once we have relation (3.1), then expression (3.3) can be seen as a function of a single variable $r$. We have adopted this approach to estimate the effective Young's moduli throughout the arterial network of the arm.

The values for $k_{1}$ and $k_{3}$ have been slightly modified from [34] (original values were $k_{1}=2.0 \times 10^{7} \mathrm{dyn} \mathrm{cm}^{-2}$

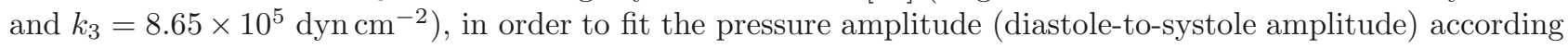
to a physiologically significant range.

\subsection{Bifurcation law}

In order to estimate the internal radius for arterial segments not found in the specialized literature, we made use of Murray's Law of bifurcations (see [42]), which is given by the expression

$$
r_{o}^{3}=r_{1}^{3}+r_{2}^{3}
$$

where $r_{o}$ denotes the radius of a parent vessel and $r_{1}, r_{2}$ the daughter radii.

\section{Anatomical And Physiological Data}

\subsection{Features of arterial connections in the arm}

The main blood supply of the arm comes from the axillary artery, whose prolonging is the brachial artery. This last one courses until the bifurcation that gives rise to the radial and ulnar arteries close to distal end of the humerus. The brachial artery and its main branches nourish the upper arm, whereas radial and ulnar arteries as well as their branches account for the blood supply to the forearm and to the hand. Previous works including arm vessels as $[4,38,43,46]$ encompass only those main four arteries plus the interosseous artery, an important branch of the ulnar artery. In turn, terminals are placed at the distal end of the radial, ulnar and interosseous arteries to simulate the resistive and compliant effects of the vascular beds beyond the ending points. Such a simplifying procedure prematurely interrupts the pressure wave propagation, changing the way in which the blood flows and yielding a loss of information concerning wave reflections. 
TABLE 1. Arterial network data. (n): number of occurrences; $L$ : lenght; ED: external diameter; $L R$ : lumen radius; BS: bibliographical sources. (*) Murray's law was employed; (**) the order of magnitude of an anatomically similar artery was used; $(* * *)$ the order of magnitude of surrounding arteries found in the bibliographical source was used.

\begin{tabular}{|c|c|c|c|c|c|}
\hline No. & Name & $L(\mathrm{~cm})$ & $E D(\mathrm{~cm})$ & $L R(\mathrm{~cm})$ & $\mathrm{BS}$ \\
\hline 1 & Axillary (1) & 12.003 & 0.560 & 0.230 & {$[3],[41],[47]$} \\
\hline 2 & Brachial (1) & 22.312 & 0.510 & 0.208 & {$[39],[45]$} \\
\hline 3 & Profunda brachial (1) & 6.221 & 0.156 & 0.058 & $(*)$ \\
\hline 4 & Thoracoacromial (1) & 0.653 & 0.379 & 0.150 & {$[4]$} \\
\hline 5 & Thoracoacromial deltoid branch (1) & 7.539 & 0.260 & 0.100 & $(*)$ \\
\hline 6 & Superior ulnar collateral artery (1) & 15.151 & 0.175 & 0.066 & {$[37]$} \\
\hline 7 & Posterior circumflex humeral (1) & 5.086 & 0.275 & 0.106 & {$[18]$} \\
\hline 8 & Anterior circumflex umeral (1) & 4.690 & 0.115 & 0.042 & {$[18]$} \\
\hline 9 & Radial collateral (1) & 11.511 & 0.125 & 0.046 & {$[13]$} \\
\hline 10 & Middle collateral (1) & 15.207 & 0.125 & 0.046 & {$[13]$} \\
\hline 11 & Middle collateral network of elbow branch (1) & 3.877 & 0.100 & 0.037 & $(* *)$ \\
\hline 12 & Inferior ulnar collateral (1) & 5.065 & 0.145 & 0.054 & {$[50]$} \\
\hline 13 & Inferior ulnar collateral network of elbow branch (1) & 2.637 & 0.100 & 0.037 & $(* *)$ \\
\hline 14 & Ulnar recurrent anterior branch (1) & 4.835 & 0.160 & 0.060 & {$[37](* * *)$} \\
\hline 15 & Ulnar recurrent posterior branch (1) & 6.148 & 0.160 & 0.060 & {$[37]$} \\
\hline 16 & Radial recurrent (1) & 6.784 & 0.200 & 0.076 & [50] \\
\hline 17 & Recurrent interosseous (1) & 5.383 & 0.100 & 0.044 & [50] \\
\hline 18 & Radial (1) & 30.216 & 0.350 & 0.138 & {$[5],[45]$} \\
\hline 19 & Ulnar (1) & 26.904 & 0.357 & 0.141 & {$[5],[45]$} \\
\hline 20 & Common interosseous (1) & 1.627 & 0.250 & 0.096 & {$[15]$} \\
\hline 21 & Anterior interosseous (1) & 22.312 & 0.070 & 0.025 & {$[40]$} \\
\hline 22 & Posterior interosseuous (1) & 23.169 & 0.180 & 0.068 & {$[40]$} \\
\hline 23 & Ulnar palmar carpal branch (1) & 1.678 & 0.065 & 0.024 & {$[23]$} \\
\hline 24 & Ulnar dorsal carpal branch (1) & 1.481 & 0.115 & 0.042 & [51] \\
\hline 25 & Ulnar deep palmar branch (1) & 1.918 & 0.260 & 0.100 & {$[45](* * *)$} \\
\hline 26 & Radial palmar carpal branch (1) & 1.578 & 0.075 & 0.027 & {$[23]$} \\
\hline 27 & Radial superficial palmar branch (1) & 4.511 & 0.170 & 0.064 & {$[5]$} \\
\hline 28 & Dorsal carpal network (2) & 3.689 & 0.065 & 0.024 & {$[35]$} \\
\hline 29 & Dorsal metacarpal (7) & 8.737 & 0.065 & 0.024 & {$[35]$} \\
\hline 30 & Princeps pollicis (1) & 4.654 & 0.193 & 0.073 & {$[5]$} \\
\hline 31 & Radial indicis (1) & 12.261 & 0.153 & 0.057 & {$[5]$} \\
\hline 32 & Princeps pollicis branch (2) & 3.960 & 0.155 & 0.058 & $(*)$ \\
\hline 33 & Radial dorsal carpal branch (1) & 0.873 & 0.135 & 0.050 & {$[51]$} \\
\hline 34 & Palmar carpal network (1) & 3.293 & 0.065 & 0.024 & {$[35](* * *)$} \\
\hline 35 & Deep palmar arch (1) & 5.349 & 0.260 & 0.100 & {$[45]$} \\
\hline 36 & Palmar metacarpal (4) & 2.941 & 0.065 & 0.024 & {$[35](* * *)$} \\
\hline 37 & Superficial palmar arch (1) & 5.175 & 0.280 & 0.108 & {$[45]$} \\
\hline 38 & Common palmar digital (4) & 3.133 & 0.175 & 0.066 & [19] \\
\hline 39 & Proper palmar digital (6) & 7.127 & 0.110 & 0.040 & {$[45]$} \\
\hline 40 & Dorsal digital (6) & 7.242 & 0.052 & 0.019 & $(*)$ \\
\hline
\end{tabular}

As already stated in the introductory section, a novel arterial topology for the arm based on anatomical and physiological considerations was built. This arterial network consists in 108 segments, where 64 of them are the arteries listed in Table 1 and the other 44 are the so-called perforator vessels. The three-dimensional structure of the arterial topology was accurately designed taking into account the pathways of vessels through muscles, connective tissue and bones. In order to carry out this task, we have used anatomical references largely adopted by the medical community. Particularly, we were preferentially guided by the Netter's Atlas of Human 
Anatomy [33], the Pocket Atlas of Human Anatomy by Feneis [16] and the Clinically Oriented Anatomy [31]. Finally, the three-dimensional vessel design was also guided by a 3D digital skeleton structure with an average height of approximately $170 \mathrm{~cm}$.

The main anastomotic sites which provide redundancy of blood supply to the network in well-defined anatomical locations have been incorporated following an average arterial connectivity. Instances of such anastomotic locations are the shoulder, the elbow and the wrist. Among the anastomoses included in this model, we point out the articular network of the elbow and the superficial and palmar archs. These two regions provide the main blood supply for the palm and fingers of the hand. Having considered these anastomoses allows the model to account for a wider range of physiological and pathophysiological scenarios of interest. Figure 3 presents several perspectives of the the vessels connectivity along the arm. Numbers are related to the data summarized in Table 1.

\subsection{Morphometrical data}

As explained before, available medical data provides arterial calibers in terms of external diameters. In addition, such studies are carried out using different populations, and some inconsistencies may arise. Thus, the external diameters which are part of the present model have been picked from the available literature such that they are consistent with surrounding neighbouring vessels. That is, a parent vessel cannot be smaller than its prolonging and bifurcating ones. In this sense the data retrieved from the literature (external diameters) is coherent.

\subsubsection{Geometrical parameters of arterial vessels}

Table 1 presents geometrical data of arterial vessels obtained from the literature and making use of (3.1), (3.2) and (3.4) to estimate the internal radii (see Sect. 3.2). The name of arteries followed the international nomenclature adopted in [16]. The number in brackets after each artery name stands for how many times this artery is repeated in the network so that the summation of all arteries must be equal to 64 segments. We point out that the lenghts of arteries resulted from the three-dimensional outline of vessels taking into account the skeletal and muscular structure and guided by anatomical references, as it was shown in Figure 3. The last column in Table 1 presents the bibliographical sources (or other criterion, when necessary) used to retrieve the external vessel diameters.

The proposed criteria rendered the spatial variation of internal radii along the arterial network of the arm shown in Figure 4, which is consistent with the data provided in Table 1.

In turn, Figure 5 displays the spatial distribution of the material parameter representing the elastin constituent along the arterial tree of the arm following the previous approach.

\subsection{Features of blood supply to vascular territories}

Source arteries are vessels whose pathway is usually parallel and sometimes close to the bone skeleton. This kind of vessels mainly accounts for the blood transport. Examples of such vessels are the brachial artery, deep brachial artery, ulnar artery and radial artery. Narrower arteries emerge from these source vessels in order to nourish both the deep fascia and the superficial fascia. Roughly speaking, the deep fascia is composed by muscles and the superficial fascia by the subcutaneous fat and the skin. According to Taylor [44], a cutaneous perforator is any vessel that branches from any source artery, goes through the deep fascia and reaches the superficial fascia. However, these cutaneous perforators achieve the superficial fascia in two different ways. In the first case, they arise from the source artery and go between the deep tissues, i.e., through the inter-muscular septums. In the second case, they pass through the muscle tissue. In the former case, they are called septocutaneous perforators and in the second case, musculocutaneous perforators. In the present work, we adopted a simplification in the sense that we do not differentiate septocutaneous and musculocutaneous perforators. So, we consider perforators just as vessels that branch from a source artery and feed a particular vascular territory, no matter if they pass between or through the muscle tissues. 

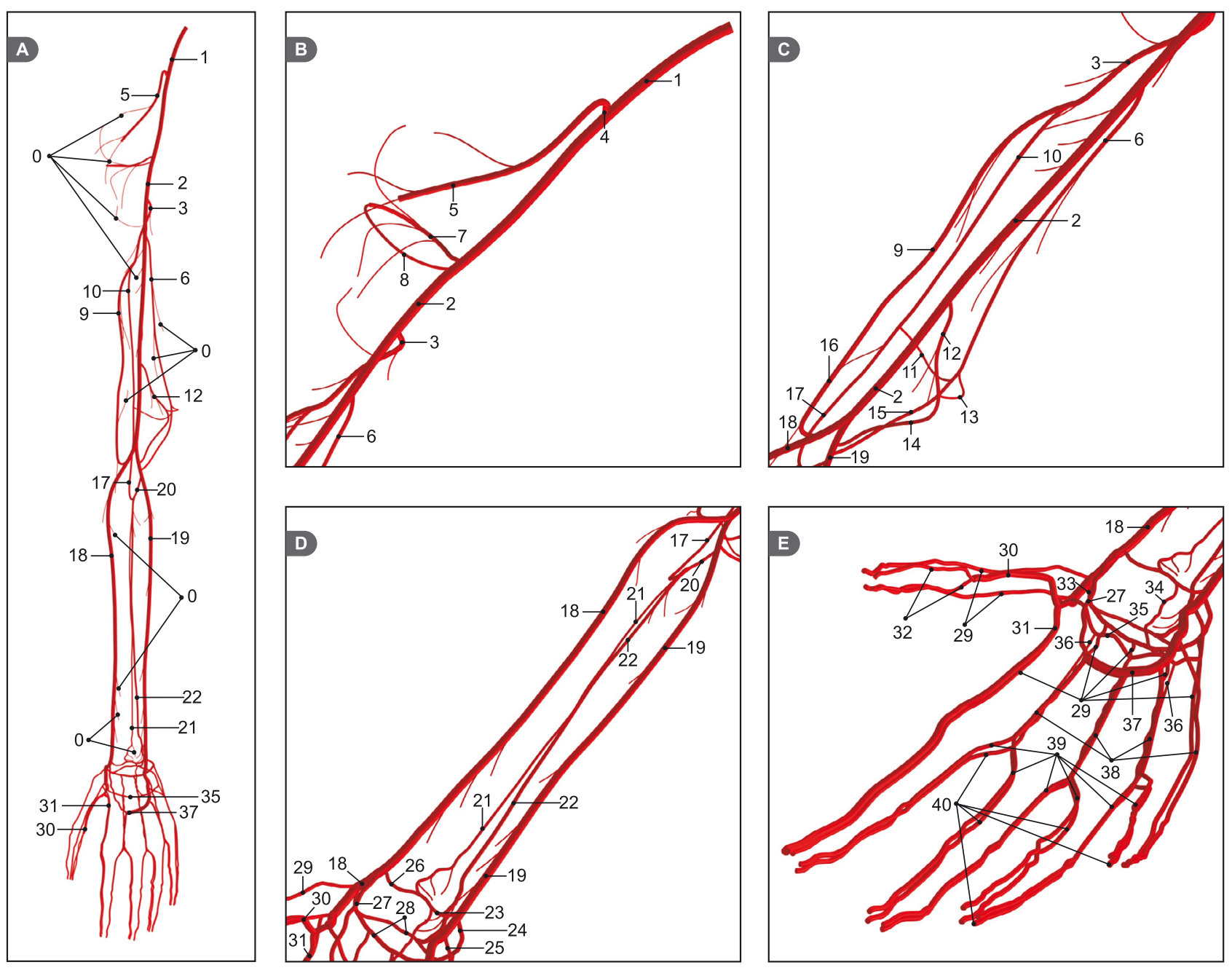

Figure 3. Anatomically detailed arterial network of the arm. A: overview of the entire network. B and C: details of the upper arm. D: details of the forearm. E: details of the wrist and hand.

On the other hand, by vascular territory we mean the portion of muscle, fat and skin that is fed by perforators that arise from some specific source vessels which play the role of blood suppliers. In other words, each vascular territory is associated to a source artery. We have based the present approach on $[32,44]$ about the placement of vascular territories and the sets of associated perforators. Particularly, the vascular territories of the arm and the corresponding source arteries are retrieved from [14]. Chen et al. have identified that perforators of the upper extremity emerge from 16 source arteries and, therefore, the upper extremity can be divided into 16 vascular territories. Figure 6 shows the division map of these 16 vascular areas of the arm.

Table 2 lists the source artery for each territory, the number of perforators per territory, the perforator diameters and the fraction of area occupied by each vascular territory. All those values in Table 2 are mean values based on [14]. Finally, we used the mapping given by [40] in order to locate places from which perforators arise from their source arteries. 


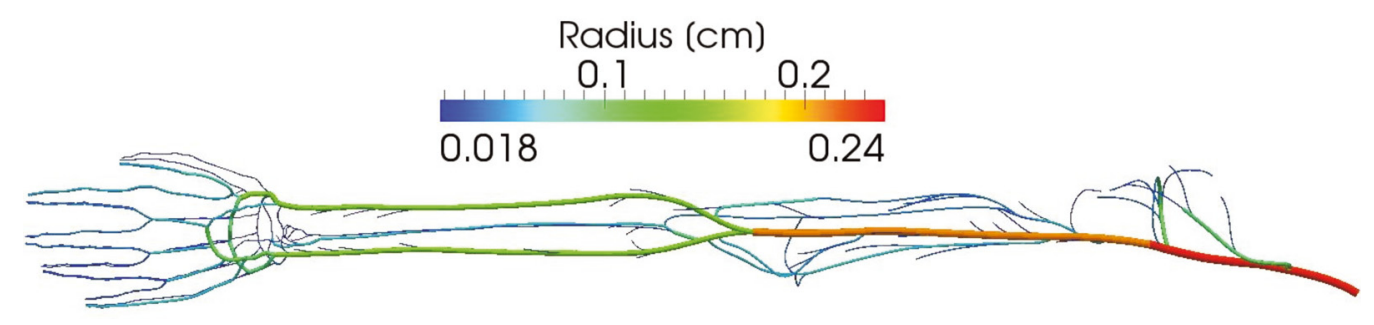

FIGURE 4. Variation of lumen radii along the arm.

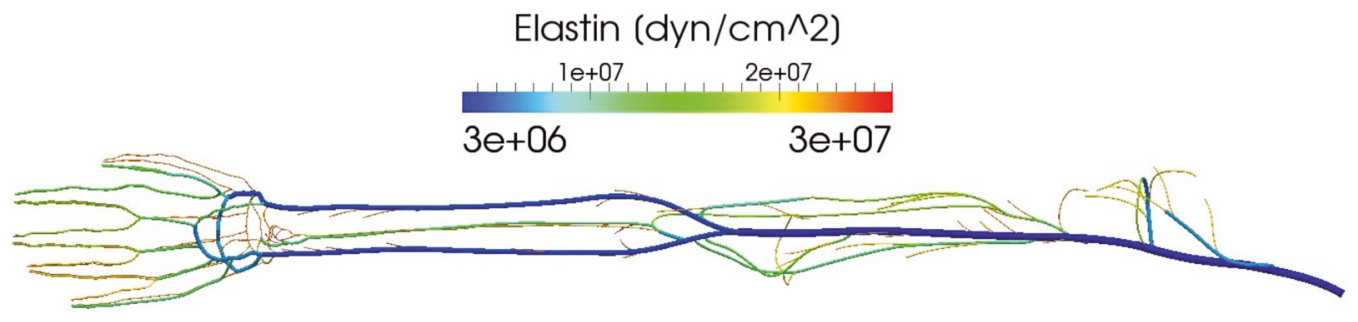

FiguRE 5. Variation of elastic moduli along the arm.

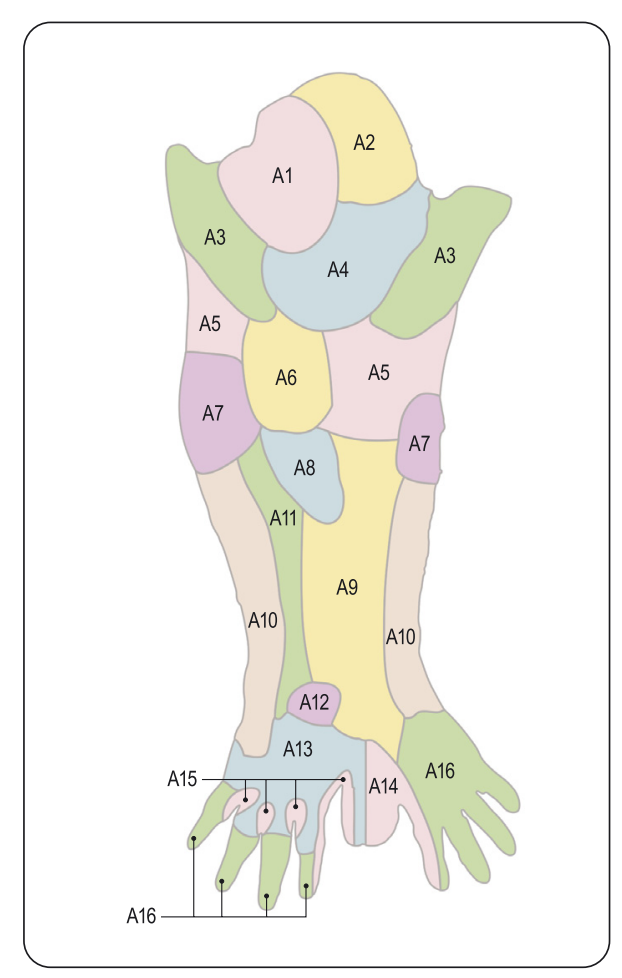

FiguRE 6. Vascular territories. Adapted from [14]. 
TABLE 2. Main data of the vascular territories. PMD: perforator mean diameter.

\begin{tabular}{lcccc}
\hline Vascular territory & Source artery & Area fraction & No. perforators & PMD (cm) \\
\hline A1 & Posterior circumflex humeral & $7.16 \%$ & 3 & 0.10 \\
A2 & Thoracoacromial (Deltoid branch) & $5.27 \%$ & 3 & 0.08 \\
A3 & Superior ulnar collateral artery & $10.86 \%$ & 2 & 0.09 \\
A4 & Profunda brachial & $7.73 \%$ & 4 & 0.07 \\
A5 & Brachial & $8.39 \%$ & 6 & 0.07 \\
A6 & Radial collateral & $4.74 \%$ & 2 & 0.10 \\
A7 & Inferior ulnar collateral & $5.93 \%$ & 2 & 0.08 \\
A8 & Radial recurrent & $2.90 \%$ & 2 & 0.07 \\
A9 & Radial & $11.93 \%$ & 5 & 0.06 \\
A10 & Ulnar & $11.29 \%$ & 7 & 0.06 \\
A11 & Posterior interosseous & $3.86 \%$ & 5 & 0.05 \\
A12 & Anterior interosseous & $0.98 \%$ & 3 & 0.05 \\
A13 & Dorsal arch and dorsal metacarpal & $5.84 \%$ & 7 & 0.05 \\
A14 & Princeps pollicis & $2.51 \%$ & 4 & 0.11 \\
A15 & Common palmar digital & $1.81 \%$ & 5 & 0.07 \\
A16 & Superficial palmar arch & $8.80 \%$ & 7 & 0.11 \\
\hline
\end{tabular}

\subsubsection{Terminal parameters}

We assessed the terminal resistances by taking into account the approximated muscle mass of each territory. For this aim, we assumed that the percentual area of each vascular territory, as listed in Table 2, provides a measure of the percentual of the related muscle mass. Then, data published in [24] allow us to evaluate the total muscular mass of the arm, while the work of Mahabir [30] allows us to estimate the muscle vascular resistance of each territory as a function of such mass. Finally, these 16 resistances were proportionally scaled in order to obtain physiological results in terms of the mean pressure. Moreover, the estimation of resistive parameters is carried out under the hypothesis that the drop pressure in the main arteries is negligible when compared with the drop pressure in terminal resistances. The validity of this hypothesis is discussed in Section 5.

In total, there are 67 terminal models distributed throughout the network. Table 3 reports the resistance values for these 67 terminals. For each vascular territory, the compact notation $T 1-T k$ means that this territory has $k$ terminals distributed along the corresponding source artery/arteries, each terminal with the resistance value given in the last column.

\section{Simulations AND Results}

In order to carry out the simulations, an inflow boundary condition is considered at point $\mathbf{A}$ shown in Figure 8. This flow rate curve corresponds to the result obtained at the proximal point of the axillary artery using an entire model of the cardiovascular system [7].

The general parameters are $\mu=0.04 \mathrm{P}, \rho=1.04 \mathrm{~g} / \mathrm{cm}^{3}$ and $P_{o}=133333 \mathrm{dyn} / \mathrm{cm}^{2}$. As said, the finite element method was used to approximate the mathematical formulation given by (2.18)-(2.19). The element size in all the simulations is $\Delta x=0.25 \mathrm{~cm}$ and the time step is $\Delta t=0.0025 \mathrm{~s}$. Three cardiac cycles have been simulated until the periodic state was reached. For this setting a satisfactory mass conservation is obtained (relative error $0.3 \%$ ).

Figure 7 shows a comparison between the pressure cuves given in [36] and the ones provided by the model developed here. Clearly, even with a very simple constitutive behavior and the inflow condition provided in [36], the model manages to reproduce the main pressure pulse features of patient records.

Figure 8 presents the inflow curve used as the proximal boundary condition (see point $\mathbf{A}$ in such figure) and the flow rate curves in six arterial districts of the arm. An important feature of the blood flow in the arm with potential impact on surgical procedures is the determination of vessel dominance in terms of blood supply to 
TABLE 3. Terminal resistances for each vascular territory.

\begin{tabular}{lccc}
\hline Vascular territory & Source artery & Terminals & $\mathcal{R}\left(\right.$ dyn $\left.\mathrm{sm}^{-2} \mathrm{ml}^{-1}\right)$ \\
\hline \hline A1 & Posterior circumflex humeral & T1-T3 & $1.416774 \mathrm{e}+006$ \\
A2 & Thoracoacromial (Deltoid branch) & T1-T3 & $2.004601 \mathrm{e}+006$ \\
A3 & Superior ulnar collateral artery & T1-T2 & $4.789409 \mathrm{e}+005$ \\
A4 & Profunda brachial & T1-T4 & $1.701231 \mathrm{e}+006$ \\
A5 & Brachial & T1-T6 & $2.259275 \mathrm{e}+006$ \\
A6 & Radial collateral & T1-T2 & $1.473350 \mathrm{e}+006$ \\
A7 & Inferior ulnar collateral & T1-T2 & $1.184357 \mathrm{e}+006$ \\
A8 & Radial recurrent & T1-T2 & $2.062935 \mathrm{e}+006$ \\
A9 & Radial & T1-T5 & $9.831755 \mathrm{e}+005$ \\
A10 & Ulnar & T1-T7 & $1.546644 \mathrm{e}+006$ \\
A11 & Posterior interosseous & T1-T5 & $4.322587 \mathrm{e}+006$ \\
A12 & Anterior interosseous & T1-T3 & $4.405444 \mathrm{e}+006$ \\
A13 & T1-T7 & $4.211632 \mathrm{e}+006$ \\
A14 & Princeps pollicis & T1-T4 & $4.435745 \mathrm{e}+006$ \\
A15 & Common palmar digital & T1-T5 & $6.301835 \mathrm{e}+006$ \\
A16 & Superficial palmar arch & T1-T7 & $2.444284 \mathrm{e}+006$ \\
\hline
\end{tabular}

the hand [45]. In the present model, the mean blood flow through the ulnar artery results in $0.7069 \mathrm{~cm}^{3} / \mathrm{s}$, while through the radial artery the mean blood flow is $0.6627 \mathrm{~cm}^{3} / \mathrm{s}$. So, the ulnar artery prevails over the radial artery as a blood supplier to the vessels in the wrist and hand, which is the most common situation encountered in practice.

As said in Section 4.3.1, the estimation of the resistive terminal parameters is strictly valid for non-viscous flow, that is, for null drop pressure in the arterial segments. Evidently, this is not the case here, although it could be considered as a good approximation. In this regard, Table 4 presents a comparison for each vascular territory of the expected blood flow, according to the data provided in Table 2, and the true blood flow which is the outcome of the numerical simulations in two scenarios: (i) steady state simulation and (ii) transient simulation. The difference observed is due to the vessel impedance which has been neglected in obtaining the data presented in Table 2. Notice that the difference between the expected blood flow and the steady state blood flow is small when compared with the differences when the flow becomes time-dependent. Making an analogy with linear models, this is the result of the additional impedance incorporated by the blood inertia and vessel distensibility.

As said in the introduction, there is a wide range of situations in medical practice which may require such a detailed model of the cardiovascular system. As mentioned in Section 4.1, the main blood supply for the forearm and the hand is provided by the radial and ulnar arteries. However, according to [45], there is a large variability in radial-ulnar blood supply dominance among subjects, and this implies in different hemodynamics in the circulation of the forearm and the hand. In most cases (55\%), the flow rate in the ulnar artery prevails over the radial one [45]. Nevertheless, in case of injury or harvest of the radial artery in certain surgical procedures ischemia of the hand may occur [23]. This is an instance for which the possibility of performing simulations taking into account different arrangements of arterial connections could lead to a better undestanding of the hemodynamic changes along the vasculature. Indeed, in the absence of the radial artery, an accurate topological description of the arterial vessels would allow to quantify the increase of the blood flow in the remaining ulnar artery as well as to know how the flow rate is distributed to the hand through the superficial and deep palmar arches. Indeed, even some neurological consequences, such as motor abnormalities in different affected areas of the hand, may arise as a consequence of lack of adequate blood flow supply to the surrounding nerves in the regions where the procedure took place [17]. The present model provides the infrastructure to make this quantification possible, being this a matter of current research. 


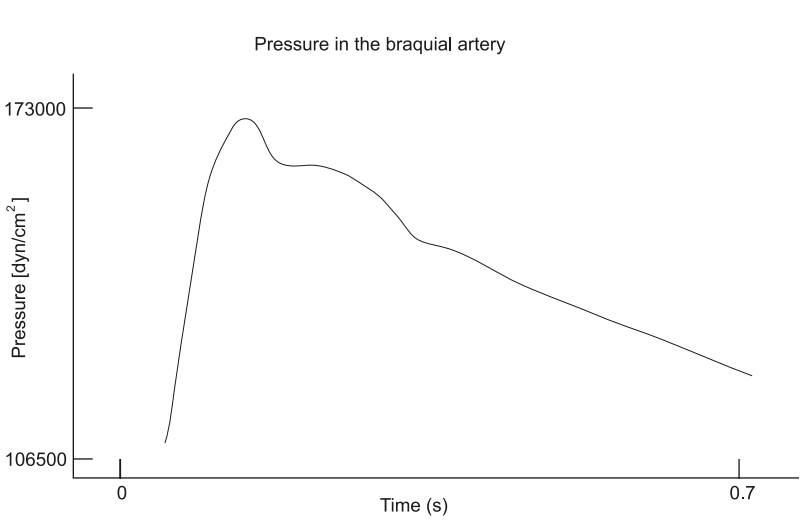

(a)

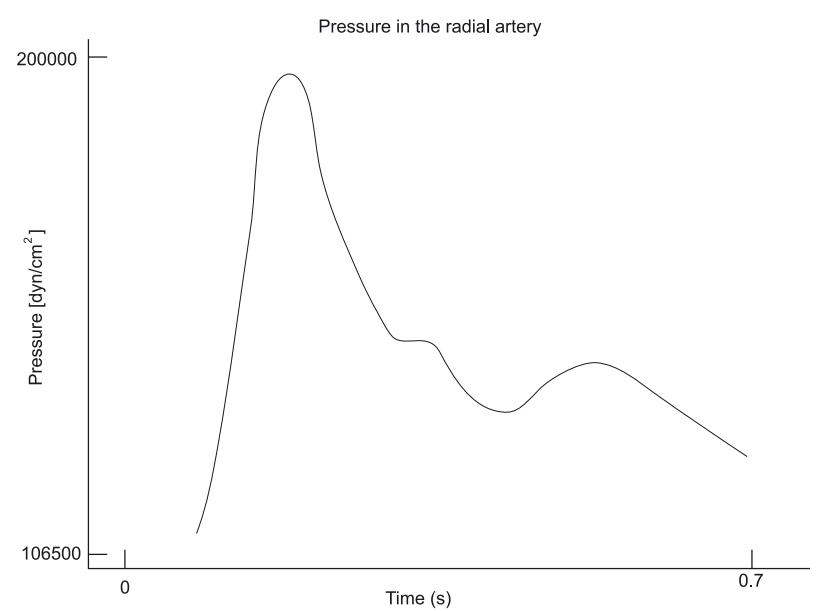

(c)

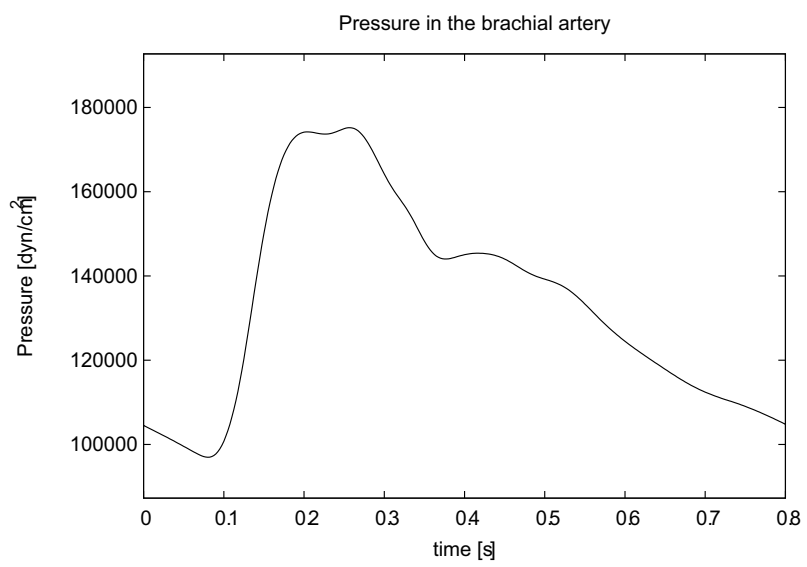

(b)

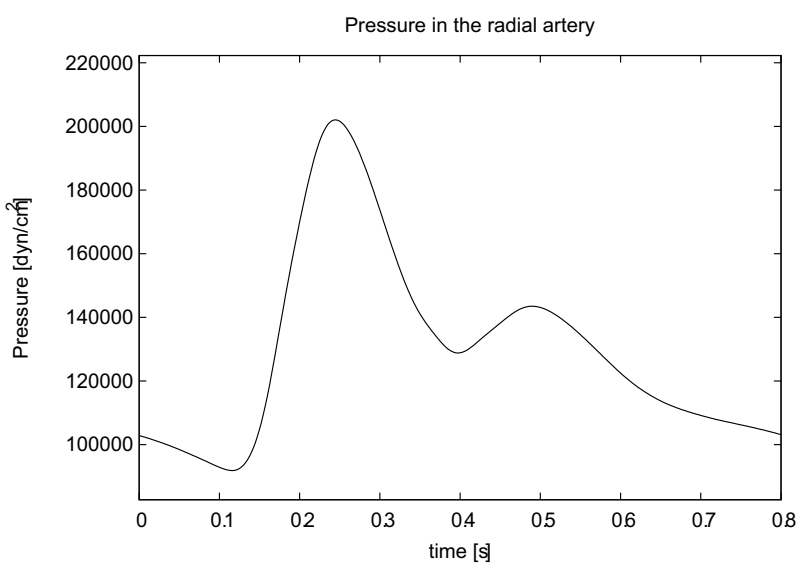

(d)

Figure 7. Pressure in the brachial artery: (a) found in [36] page 184 and (b) provided by the model. Pressure in the radial artery: (c) found in [36] page 184 and (d) provided by the model.

\section{Dimensionally-heterogeneous modeling}

Dimensionally-heterogeneous modeling has been applied in a wide variety of applications in order to provide insight on the local blood flow patterns and its relation to global hemodynamic environments [9-11,22, 26, 46]. In this section we apply the detailed model of the arterial network of the arm developed in Sections 3 and 4 to study the blood flow in a branching site.

The 3D model matches the geometrical and mechanical parameters of the 1D model in which it is embedded. The bifurcation under analysis corresponds to the branching of the brachial artery into the ulnar and radial arteries. Also, the ulnar recurrent anterior and posterior branches, as well as the branches joining with the common interosseous and the radial recurrent vessels are present (see Fig. 3 and Tab. 1 for a reference to these vessels).

Regarding the description of the numerical methodologies to address the solution of the 3D Navier-Stokes equations in compliant domains and its coupling with the $1 \mathrm{D}$ model the reader is referred to $[7,8,29]$. Here we provide a brief account of the domain decomposition strategy employed to iteratively couple the 1D and 

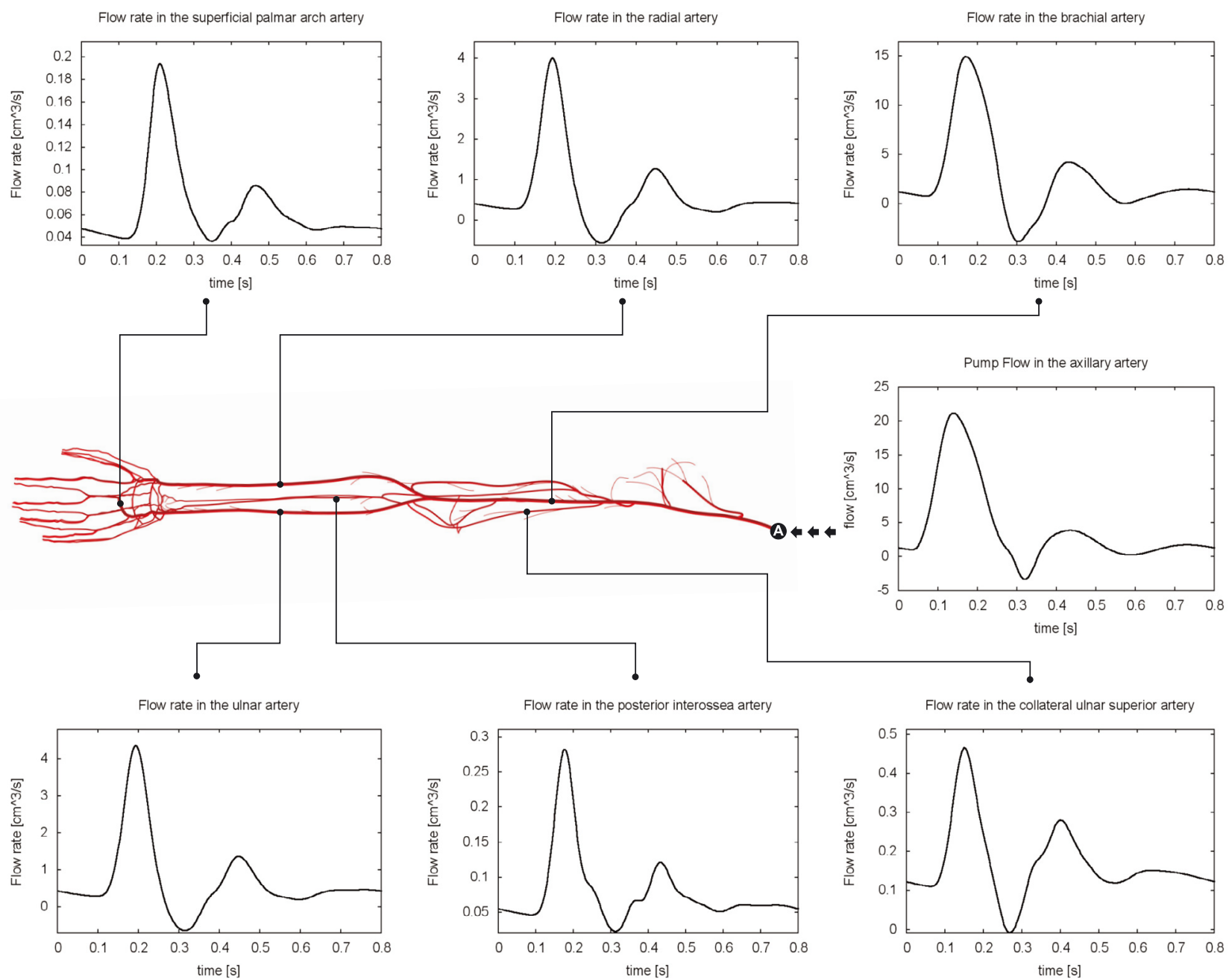

FigURE 8. Blood flow rate at several locations in the arterial vasculature of the arm.

3D models. Figure 10 shows the embedding of the 3D geometry within the 1D model presented in Section 4 . The decomposition approach described in [29] amounts to define at each interface the coupling degrees of freedom, namely flow rate and pressure $\left(Q_{i}, P_{i}\right), i=1, \ldots, 7$ (notice that we have 7 coupling interfaces). For the numbering of the coupling interfaces see also Figure 10.

The basic idea is to conceive both models as black-boxes which receive some input data at the coupling interfaces and give back an output result at the same points. In the present work, the input data for both models is the set of pressure values at the coupling interfaces $\mathbf{P}=\left(P_{1}, \ldots, P_{7}\right)$, while the output is the flow rate $\mathbf{Q}=\left(Q_{1}, \ldots, Q_{7}\right)$. It is worth noting that it has been pointed out more advantageous properties, regarding energy stability of the coupled system, of imposing the dynamic pressure to the 3D flow model [21]. Nevertheless, for the cases and flow regimes analyzed in the present work imposing the static pressure did not cause any instability issue. Then, the 1D model receives the $\mathbf{P}$ as boundary condition and gives back the flow rate $\mathbf{Q}$. This relation can be written in compact abstract form as

$$
\mathbf{Q}=\mathcal{F}_{1 D}(\mathbf{P}),
$$


TABLE 4. Vascular territory flow comparison. EBF: expected blood flow. SBF: steady state blood flow. TBF: transient blood flow.

\begin{tabular}{lccc}
\hline Vascular territory & EBF $\left(\mathrm{cm}^{3} / \mathrm{s}\right)$ & SBF $\left(\mathrm{cm}^{3} / \mathrm{s}\right)$ & TBF $\left(\mathrm{cm}^{3} / \mathrm{s}\right)$ \\
\hline A1 & 0.257910 & 0.269581 & 0.286350 \\
A2 & 0.182280 & 0.187478 & 0.202567 \\
A3 & 0.508622 & 0.502985 & 0.528555 \\
A4 & 0.286380 & 0.297155 & 0.300144 \\
A5 & 0.323466 & 0.334631 & 0.357215 \\
A6 & 0.165338 & 0.169642 & 0.161771 \\
A7 & 0.205682 & 0.207770 & 0.220974 \\
A8 & 0.118084 & 0.119136 & 0.127546 \\
A9 & 0.619420 & 0.623865 & 0.664388 \\
A10 & 0.551257 & 0.554456 & 0.590978 \\
A11 & 0.140890 & 0.139425 & 0.146602 \\
A12 & 0.082944 & 0.078748 & 0.071208 \\
A13 & 0.202440 & 0.180975 & 0.065835 \\
A14 & 0.109836 & 0.107375 & 0.102361 \\
A15 & 0.096640 & 0.093751 & 0.077699 \\
A16 & 0.348817 & 0.340065 & 0.329007 \\
\hline
\end{tabular}

where $\mathcal{F}_{1 D}$ represents the abstract operator which stands for the solution of the $1 \mathrm{D}$ equations presented in Section 1. In turn, for the 3D model it is necessary firstly to perform an extension of the point-wise data $\mathbf{P}$ in order to meet the $3 \mathrm{D}$ boundary data needed at the coupling boundaries $\Gamma_{i}, i=1, \ldots, 7$. This extension is denoted by $\mathrm{E}(\mathbf{P})$, and in the present work consists in the trivial extension by constant of $P_{i}$ to the 3D boundary $\Gamma_{i}, i=1, \ldots, 7$. Then, from the $3 \mathrm{D}$ model the flow rate $\mathbf{Q}$ is retrieved as follows

$$
\mathbf{Q}=\operatorname{R}\left(\mathcal{F}_{3 D}(\mathrm{E}(\mathbf{P}))\right)
$$

where $\mathcal{F}_{3 D}$ is the abstract operator that represents the solution of the Navier-Stokes equations, and $\mathrm{R}$ is a restriction operator that integrates the $3 \mathrm{D}$ velocity profile $\mathbf{v}_{i}$ over $\Gamma_{i}$ in the direction of the normal $\mathbf{n}_{i}$, and gives the corresponding flow rate $Q_{i}, i=1, \ldots, 7$. Clearly, we have to look for the solution of the following system of nonlinear equations

$$
\mathcal{R}(\mathbf{Q}, \mathbf{P})=\left(\begin{array}{l}
\mathcal{R}_{1 D}(\mathbf{Q}, \mathbf{P}) \\
\mathcal{R}_{3 D}(\mathbf{Q}, \mathbf{P})
\end{array}\right)=\left(\begin{array}{c}
\mathbf{Q}-\mathcal{F}_{1 D}(\mathbf{P}) \\
\mathbf{Q}-\mathrm{R}\left(\mathcal{F}_{3 D}(\mathrm{E}(\mathbf{P}))\right)
\end{array}\right)=0
$$

Operators $\mathcal{F}_{1 D}$ and $\mathcal{F}_{3 D}$ involved in (6.3) are time-dependent and non-linear. As aforesaid, these nonlinearities are treated using fixed point iterations. Therefore, in order to solve this problem using any iterative method, a time discretization and posterior linearization are in order. For the 1D problem the numerical approach has been explained in Section 1. For the 3D problem we use an implicit formulation for the velocity-pressure variables with a Crank-Nicolson time-discretization and a fixed point method for the linearization of the convective term. Thus, (6.3) is written for each time step as follows

$$
\mathcal{R}^{n+1}\left(\mathbf{Q}^{n+1}, \mathbf{P}^{n+1}\right)=\left(\begin{array}{c}
\mathcal{R}_{1 D}^{n+1}\left(\mathbf{Q}^{n+1}, \mathbf{P}^{n+1}\right) \\
\mathcal{R}_{3 D}^{n+1}\left(\mathbf{Q}^{n+1}, \mathbf{P}^{n+1}\right)
\end{array}\right)=\left(\begin{array}{c}
\mathbf{Q}^{n+1}-\mathcal{F}_{1 D}^{n+1}\left(\mathbf{P}^{n+1}\right) \\
\mathbf{Q}^{n+1}-\mathrm{R}\left(\mathcal{F}_{3 D}^{n+1}\left(\mathrm{E}\left(\mathbf{P}^{n+1}\right)\right)\right)
\end{array}\right)=0 \quad n=0,1, \ldots
$$

The fact that we put $\mathcal{F}_{1 D}^{n+1}$ and $\mathcal{F}_{3 D}^{n+1}$ implies that these operators are nonlinear. In this work, we make use of the Broyden method to solve this system of equations at each time step. Calling $\mathbf{x}=(\mathbf{Q}, \mathbf{P})$, the algorithm is the following. 


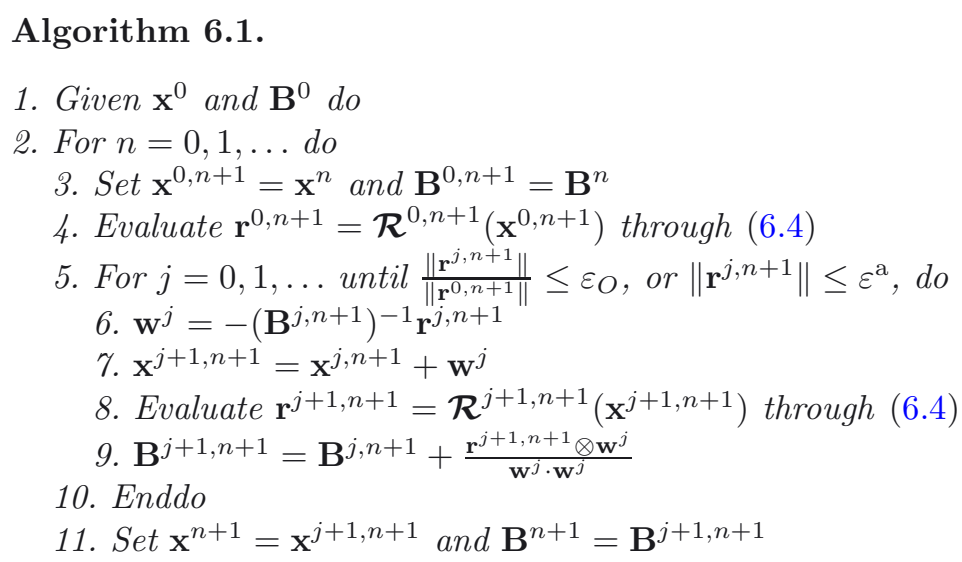

12. Enddo

The initial matrix $\mathbf{B}^{0}$ is the Jacobian of the system computed through finite differences, that is

$$
B_{k l}^{0}=\frac{\mathcal{R}_{k}^{0}\left(x_{l}^{0}+\delta x_{l}^{0}\right)-\mathcal{R}_{k}^{0}\left(x_{l}^{0}\right)}{\delta x_{l}^{0}} \quad k, l=1, \ldots, 14 .
$$

where $\mathcal{R}_{k}^{0}(\cdot)$ is the $k$ th component of $\mathcal{R}^{0}(\cdot)$ and $\delta x_{l}^{0}=\epsilon x_{l}^{0}, l=1, \ldots, 14$, with $\epsilon$ small enough.

Unlike classical Gauss-Seidel algorithms used in previous works to deal with the 1D-3D coupling, the Broyden algorithm provides a flexible and robust approach to solve (6.3). In fact, the hierarchical nature of Gauss-Seidel methods poses restriction on the choice of the input data for the models. This means, for instance, that solving the system (6.3) in which pressure $\mathbf{P}$ is imposed at both models would not have been a plausible approach with those strategies.

In this case we run three cardiac cycles and employed a time step $\Delta t=0.001 \mathrm{~s}$. The results presented below correspond to the last heart beat. Figure 9 shows that the number of iterations the coupling algorithm takes to reach convergence at each time step ranges from 2 to 6 (mean 3.2), which is an excellent performance in view of the number of unknowns in the system ( 7 coupling interfaces, 14 interface unknowns).

Figure 10 presents the results in terms of pressure and flow rate at the coupling interfaces. Positive flow rate is always defined in the proximal-to-distal direction. Notice the change in the pressure wave form as a result of the vessel distensibility, and recall that blood flow distribution is mostly dictated by resistive parameters representing the vascular territories.

In Figure 11 the pressure field in the 3D geometry is presented at four time instants in the cardiac cycle. Observe the inversion of the pressure gradient right after systole. This change in the direction of the pressure gradient is responsible for the presence of Womersley-like velocity profiles during diastole, as seen in the same figure. Also, observe the highly complex pattern of blood flow at the posterior and anterior recurrent ulnar branches, a common feature during the entire cardiac cycle. This feature is absolutely neglected when making use of 1D modeling, which may cause an impact on the flow rate wave form and the mean flow direction as observed in Figure 10. In such figure, it is observed that the mean flow rate through these ulnar branches is negative (outgoing the 3D bifurcation), pointing out that these are natural anastomotic branches present in the elbow which form a graft with the recurrent interosseous artery.

\section{Conclusions}

In this work, an highly detailed model of the vasculature of the arm has been developed. Calibration of geometrical and material parameters has been accomplished following anatomical and physiological considerations. In addition, the estimation of resistive parameters in terminal models accounting for peripheral beds has been 


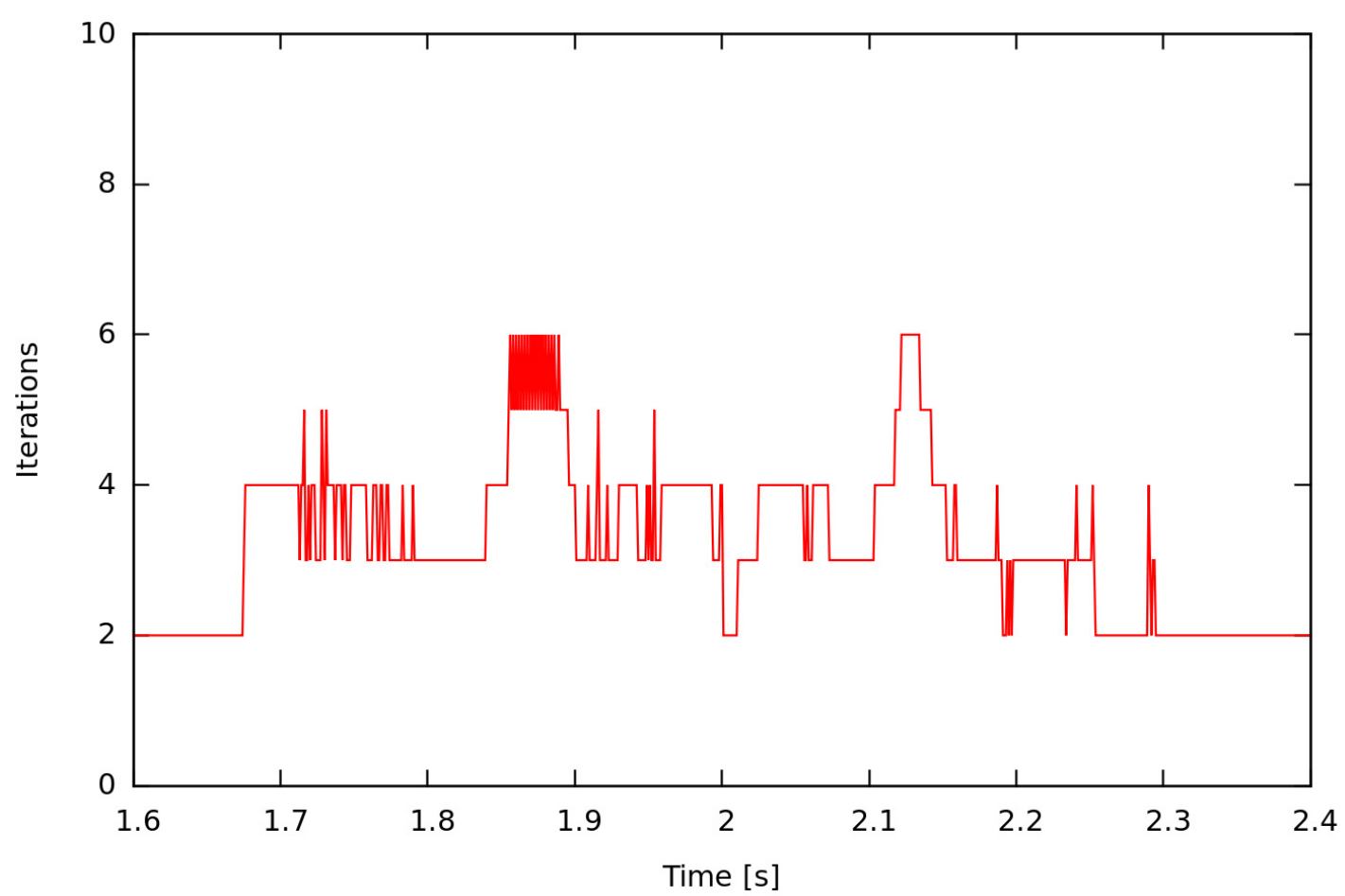

Figure 9. Performance of Broyden algorithm. Number of iterations to reach convergence at each time step throughout the third cardiac cycle.

achieved according to the physiological characteristics of vascular territories. Pressure pulse waves obtained from numerical simulations are in agreement with patient records. In view of the highly detailed anatomical description of the arterial topology, this kind of model infrastructure serves as a laboratory for simulating reconstructive surgery procedures, among others. Indeed, its application to the outcomes of radial harvesting for coronary revascularization is being a matter of current research. Finally, the possibility of performing dimensionally-heterogeneous modeling with the aim of analyzing local hemodynamics in places of interest can be further explored in detail. In this work, its application to the study of the blood flow at the brachial bifurcation leading to the radial and ulnar vessels has been presented.

It is worthwhile to say that not only applications, but also computational/numerical challenges are triggered by this kind of highly detailed model/datasets. For instance, the flow rate features several orders of magnitude throughout the entire network. This poses some difficulties in view of the conditioning of the system of non-linear equations. Indeed, in the present model, fixed point iterations per time step range from 20 to 30 throughout the entire cardiac beat. Hence, more efficient numerical algorithms (e.g. based on velocity-area unknowns) as well as acceleration techniques to deal with this problem will certainly improve the realization of numerical simulations in a suitable period of time for the medical community.

The simplified approach considered in this work towards setting the peripheral resistances is certainly a point to be improved. As a result of this simplification it was observed in the results that the transient blood flow differed from the expected blood flow. A strategy based on inverse estimation of peripheral resistances would constitute a formal and robust approach to solve this problem. Moreover, devising robust numerical algorithms for properly dealing with viscoelasticity is also to be addressed in the short term in order to improve the features of the model. At last, but not least, we should mention the possibility of performing in-silico validation of the $1 \mathrm{D}$ simulations using 3D models for the entire topology of the arm. Solving the present network using the 3D 

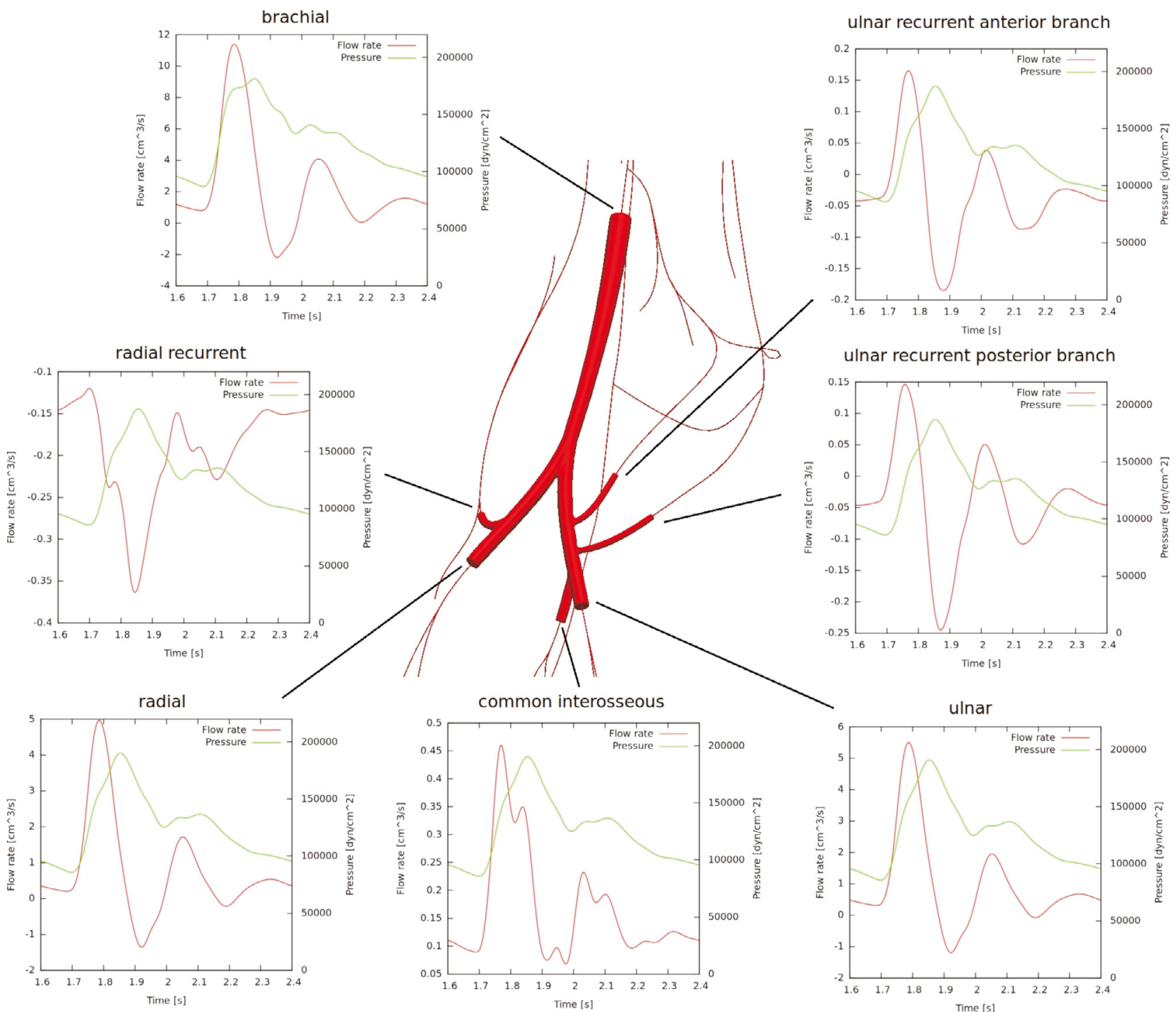

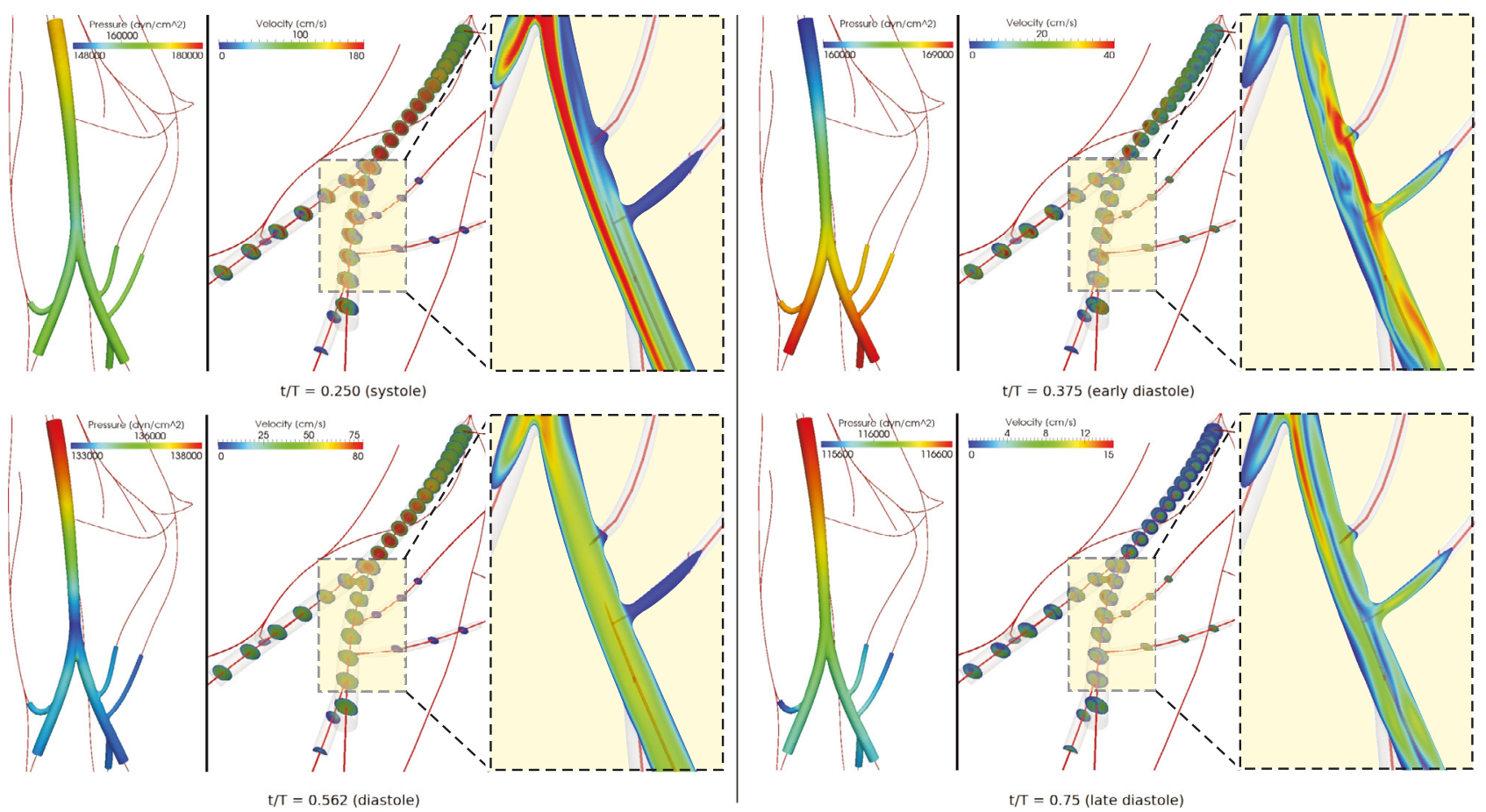

$\mathrm{t} / \mathrm{T}=0.75$ (late diastole)

FiguRE 11. Pressure and velocity profiles in several slices at different time instants during the cardiac cycle. Also, a detail of the velocity field is shown at the ulnar artery and its recurrent branches.

Acknowledgements. This work was partially supported by the Brazilian agencies CNPq and FAPERJ. The support of these agencies is gratefully acknowledged. The authors would like to acknowledge Dr. Marco Aurélio da Fonseca Passos from the Department of Anatomy of the Faculty of Medicine in Petrópolis for his support.

\section{REFERENCES}

[1] J. Alastruey, A.W. Khir, K.S. Matthys, P. Segers, S.J. Sherwin, P.R. Verdonck, K.H. Parker and J. Peiró, Pulse wave propagation in a model human arterial network: Assessment of 1-D visco-elastic simulations against in vitro measurements. $J$. Biomech. 44 (2011) 2250-8.

[2] J. Alastruey, K.H. Parker, J. Peiró, S.M. Byrd and S.J. Sherwin, Modelling the circle of Willis to assess the effects of anatomical variations and occlusions on cerebral flows. J. Biomech. 40 (2007) 1794-805.

[3] B.H. Amundsen, U. Wisloff, J. Helgerud, J. Hoff and S.A. Slordahl, Ultrasound recorded axillary artery blood flow during elbow-flexion exercise. Medicine and science in sports and exercise 34 (2002) 1288-93.

[4] A.P. Avolio, Multi-branched model of the human arterial system. Medical Biolog. Engrg. Comp. 18 (1980) $709-18$.

[5] O. Bilge, Y. Pinar, M.A. Ozer and F. Gövsa, A morphometric study on the superficial palmar arch of the hand. Surg. Radiol. Anat.: SRA 28 (2006) 343-50.

[6] P.J. Blanco and R.A. Feijóo, The role of the variational formulation in the dimensionally-heterogeneous modelling of the human cardiovascular system, in Modeling of Physiological Flows, edited by H. Ambrosi, A. Quarteroni and G. Rozza. Springer, Italy (2012) 251-288.

[7] P.J. Blanco, R.A. Feijóo and S.A. Urquiza, A unified variational approach for coupling 3D-1D models and its blood flow applications. Comput. Methods Appl. Mech. Engrg. 196 (2007) 4391-4410.

[8] P.J. Blanco, J.S. Leiva, R.A. Feijóo and G.S. Buscaglia, Black-box decomposition approach for computational hemodynamics: One-dimensional models. Comput. Methods Appl. Mech. Engrg. 200138 (2011) 9-1405.

[9] P.J. Blanco, M.R. Pivello, S.A. Urquiza and R.A. Feijóo, On the potentialities of 3D-1D coupled models in hemodynamics simulations. J. Biomech. 42 (2009) 919-930. 
[10] P.J. Blanco, P.R. Trenhago, L.G. Fernandes and R.A. Feijóo, On the integration of the baroreflex control mechanism in a heterogeneous model of the cardiovascular system. Int. J. Numer. Methods Biomed. Engng. 28 (2012) $412-433$.

[11] P.J. Blanco, S.A. Urquiza and R.A. Feijóo, Assessing the influence of heart rate in local hemodynamics through coupled 3D-1D-0D models. Int. J. Numer. Methods Biomed. Engng. 26 (2010) 890-903.

[12] P.J. Blanco, S.M. Watanabe and R.A. Feijóo, Identification of vascular territory resistances in one-dimensional hemodynamics simulations. J. Biomech. 45 (2012) 2066-2073.

[13] V. Casoli, E. Kostopoulos, P. Pélissier, P. Caix, D. Martin and J. Baudet, The middle collateral artery: anatomic basis for the extreme lateral arm flap. Surg. Radiol. Anat.: SRA 26 (2004) 172-7.

[14] S. Chen, D. Xu, M. Tang, H. Ding, W. Sheng and T. Peng, Measurement and analysis of the perforator arteries in upper extremity for the flap design. Surg. Radiol. Anat.: SRA 31 687-93 (2009).

[15] H. Claassen, O. Schmitt, D. Werner, W. Schareck, J.C. Kröger and A. Wree, Superficial arm arteries revisited: Brother and sister with absent radial pulse. Annals of anatomy = Anatomischer Anzeiger: official organ of the Anatomische Gesellschaft 192 (2010) 151-5.

[16] W. Dauber, Pocket Atlas of Human Anatomy by Feneis. Thieme, 5th edition (2007).

[17] T.A. Denton, L. Trento, M. Cohen, R.M. Kass, C. Blanche, S. Raissi, W. Cheng, G.P. Fontana and A. Trento, Radial artery harvesting for coronary bypass operations: neurologic complications and their potential mechanisms. The Journal of thoracic and cardiovascular surgery 121 (2001) 951-6.

[18] F. Duparc, J.M. Muller and P. Fréger, Arterial blood supply of the proximal humeral epiphysis. Surg. Radiol. Anat.: SRA 23 (2001) 185-90.

[19] V.P.S. Fazan, C.T. Borges, J.H. Da Silva, A.G. Caetano and O.A.R. Filho, Superficial palmar arch: an arterial diameter study. J. Anat. 204 (2004) 307-11.

[20] L. Formaggia, D. Lamponi and A. Quarteroni, One-dimensional models for blood flow in arteries. J. Engrg Math. 47 (2003) $251-276$.

[21] L. Formaggia, A. Moura and F. Nobile, On the stability of the coupling of 3D and 1D fluid-structure interaction models for blood flow simulations. ESAIM: M2AN 41 (2007) 743-769.

[22] L. Formaggia, F. Nobile, A. Quarteroni and A. Veneziani, Computing and Visualization in Science Regular article Multiscale modelling of the circulatory system: a preliminary analysis. 83 (1999) 75-83.

[23] M. Haerle, F. Tonagel and H.E. Schaller, Collateral arterial pathways in the forearm. Surg. Radiol. Anat.: SRA 26 (2004) 208-11.

[24] K.R.S. Holzbaur, W.M. Murray, G.E. Gold and S.L. Delp, Upper limb muscle volumes in adult subjects. J. Biomech. (2007) $742-749$.

[25] T.J.R. Hughes and J. Lubliner, On the one-dimensional theory of blood flow in the larger vessels. Math. Biosci. 18 (1973) $161-170$.

[26] H.J. Kim, I.E. Vignon-Clementel, C.A. Figueroa, J.F. LaDisa, K.E. Jansen, J.A. Feinstein and C.A. Taylor, On coupling a lumped parameter heart model and a three-dimensional finite element aorta model 37 (2009) 2153-2169.

[27] K. Knobloch, S. Tomaszek, A. Lichtenberg, M. Karck and A. Haverich, Long-term palmar microcirculation after radial artery harvesting: an observational study. The Annal. Thorac. Surg. 81 (2006) 1700-7.

[28] C.A.D. Leguy, E.M.H. Bosboom, A.S.Z. Belloum, A.P.G. Hoeks and F.N. van de Vosse, Global sensitivity analysis of a wave propagation model for arm arteries. Medical Engrg. Phys. 33 (2011) 1008-16.

[29] J.S. Leiva, P.J. Blanco and G.C. Buscaglia, Partitioned analysis for dimensionally-heterogeneous hydraulic networks. SIAM Multiscale Model. Simulat. 9 (2011) 872-903.

[30] R.C. Mahabir, J.S. Williamson, N.J. Carr and D.J. Courtemanche, Vascular Resistance in Human Muscle Flaps. Annal. Plast. Surg. 47 (2001) 148-152.

[31] K.L. Moore, A.F. Dalley and A.M.R. Agur, Clinically Oriented Anatomy. Wolters Kluwer, 6th edition (2010).

[32] S.F. Morris, M. Tang, K. Almutari, C. Geddes and D. Yang, The anatomic basis of perforator flaps. Clinics in plastic surgery 37 (2010) 553-70.

[33] F.H. Netter, Atlas of Human Anatomy. Elsevier, 5th edition (2011).

[34] M.S. Olufsen, Structured tree outflow condition for blood flow in larger systemic arteries. Amer. J. Phys. 276 (1999) H257-68.

[35] S. Omokawa, Y. Tanaka, J. Ryu and V.L. Kish, The anatomical basis for reverse first to fifth dorsal metacarpal arterial flaps. J. Hand Surgery (Edinburgh, Scotland) 30 (2005) 40-4.

[36] M.F. O'Rourke and W.W. Nichols, McDonald's Blood Flow in Arteries - Theoretical, Experimental and Clinical Principles. Arnold, 4th edition (1998).

[37] C.D. Prevel, H.S. Matloub, Z. Ye, J.R. Sanger and N.J. Yousif, The extrinsic blood supply of the ulnar nerve at the elbow: an anatomic study. The J. Hand Surgery 18 (1993) 433-8.

[38] P. Reymond, F. Merenda, F. Perren, D. Rüfenacht and N. Stergiopulos, Validation of a one-dimensional model of the systemic arterial tree. Amer. J. Physiol. Heart circulatory Physiol. 297 (2009) H208-22.

[39] A.G. Royse, G.S. Chang, D.M. Nicholas and C.F. Royse, No late ulnar artery atheroma after radial artery harvest for coronary artery bypass surgery. The Annal. Thoracic Surgery $\mathbf{8 5}$ (2008) 891-4.

[40] M. Sauerbier and F. Unglaub, Perforator flaps in the upper extremity. Clinics in plastic Surg. 37 (2010) 667-76.

[41] I. Schafhalterzoppoth and A. Gray, The Musculocutaneous Nerve: Ultrasound Appearance for Peripheral Nerve Block. Regional Anesthesia and Pain Medicine 30 (2005) 385-390. 
[42] T.F. Sherman, On Connecting Large Vessels to Small The Meaning of Murray's Law. J. General Physiol. (1981).

[43] N. Stergiopulos, D.F. Young and T.R. Rogge, Computer simulation of arterial flow with applications to arterial and aortic stenoses. J. Biomech. 25 (1992) 1477-1488.

[44] G. Taylor, The angiosomes of the body and their supply to perforator flaps. Clin. Plast. Surg. 30 (2003) 331-342.

[45] S. Trager, M. Pignataro, J. Anderson and J.M. Kleinert, Color flow Doppler: imaging the upper extremity. J. Hand Surg. 18 (1993) 621-5.

[46] S.A. Urquiza, P.J. Blanco, M.J. Vénere and R.A. Feijóo, Multidimensional modelling for the carotid artery blood flow. Comput. Methods Appl. Mech. Engrg. 195 (2006) 4002-4017.

[47] G. Walther, S. Nottin, M. Dauzat and P. Obert, Femoral and axillary ultrasound blood flow during exercise: a methodological study. Med. Sci. Sports Exerc. 38 (2006) 1353.

[48] J.J. Wang and K.H. Parker, Wave propagation in a model of the arterial circulation. J. Biomech. 37 (2004) 457-70.

[49] G. Wavreille, J. Bricout, S. Mouliade, S. Lemoine, G. Prodhomme, P. Khanchandani, C. Chantelot and C. Fontaine, Anatomical bases of the free posterior brachial fascial flap. Surg. Radiol. Anat.: SRA 32 (2010) 393-9.

[50] G. Wavreille, C. Dos Remedios, C. Chantelot, M. Limousin and C. Fontaine, Anatomic bases of vascularized elbow joint harvesting to achieve vascularized allograft. Surg. Radiol. Anat.: SRA 28 (2006) 498-510.

[51] F.H. Zhang, S.G. Topp, W.J. Zhang, H.P. Zheng and F. Zhang, Anatomic study of distally based pedicle compound flaps with nutrient vessels of the cutaneous nerves and superficial veins of the forearm. Microsurgery 26 (2006) 373-385. 